\title{
A NEW FRAMEWORK FOR RECONSTRUCTION OF IMAGES FROM BOUNDARIES*
}

\author{
D. RAVI ${ }^{\dagger}$ AND Y. V. VENKATESH ${ }^{\dagger}$
}

\begin{abstract}
The problem of reconstructing images from boundaries has been studied in the literature chiefly as one based on the information from zero crossings. In contrast, we formulate the problem within the framework of approximation theory, and we propose a representation scheme involving (i) the points arbitrarily near the boundaries and (ii) the gradient information at these points. We show that the representation scheme is complete and stable under reasonable conditions. Further, we develop an efficient reconstruction mechanism, using the concept of a regularized solution, in which we penalize a candidate solution in proportion to its departure from a model which resembles the original in shape but is simple to construct. Finally, we demonstrate the effectiveness of the cost functional with the help of experimental results, using a representation in which the number of data points is much less than the number of pixels in the original image.
\end{abstract}

Key words. computer vision, boundary detection, image gradient, regularization, stable image reconstruction, inverse problems, zero crossings

AMS subject classification. 94A12

PII. S0036139998343861

1. Introduction. The goal of computer vision is to construct a machine which can understand and interpret visual information much as we humans do. To this end, we need to develop an appropriate framework for analyzing two-dimensional signals. Marr [1] suggested extraction of points (in the given image) where sudden changes of intensity (gray values) take place. These points, which are identified with those on the boundaries of objects in the scene [1], lead to the creation of what Marr calls a primal sketch.

In order that the representation in terms of boundaries or contours be useful, we must make sure that there is a one-to-one correspondence between an image and its representation. Now the problem is to establish conditions which guarantee that the representation is faithful (i.e., that there is indeed a one-to-one correspondence between the image and its representation). Also, when we perform operations on the representation (which we can call the transformed version) of the image, we need to make sure that small changes in the transformed domain correspond to small changes in the original image. This calls for the continuity of the mapping from the representation to the original. Apart from the theoretical interest in knowing whether or not a particular representation is faithful and stable, a practical requirement is the reconstruction of the original image in a stable manner from its representation. Generically referred to as reconstruction from partial information, this encompasses a wide range of problems. An instance of this in computer vision is the problem of reconstruction from modulus maxima (RMM) of directional derivatives which we address here.

We adopt the following notation:

- $d$ is a positive integer.

*Received by the editors August 24, 1998; accepted for publication (in revised form) September 1, 1999; published electronically May 31, 2000.

http://www.siam.org/journals/siap/60-6/34386.html

${ }^{\dagger}$ Computer Vision and Artificial Intelligence Laboratory, Department of Electrical Engineering, Indian Institute of Science, Bangalore 560 012, India (ravi@ee.iisc.ernet.in, yvvele@ee.iisc.ernet.in). 
- For $x, y \in R^{d}, x \cdot y$ stands for the standard inner product of $x$ and $y ;|x|$ stands for the Euclidean norm.

- For $x \in R^{d}$ and $r>0, B(x ; r)$ stands for the open ball of radius $r$ centered at $x$.

- For $f, g \in \mathcal{L}^{2}\left(R^{d}\right),\langle f, g\rangle$ stands for the inner product of $f$ and $g$, and $\|f\|$ for the norm of $f$.

- For a differentiable function $f: R^{d} \rightarrow R, D_{i} f$ stands for the partial derivative of $f$ with respect to the $i$ th coordinate. Similarly, if $f$ is twice differentiable, $D_{i j} f$ stands for the partial derivative with respect to the $i$ th and $j$ th coordinates. For a unit vector $\mathbf{u}$ of $R^{d}$ the directional derivative, $\partial_{\mathbf{u}} f$, is defined as

$$
\begin{aligned}
\partial_{\mathbf{u}} f & =\mathbf{u} \cdot \nabla f \\
& =\sum_{i} u_{i} D_{i} f .
\end{aligned}
$$

- $\theta(x), x \in R^{d}$ stands for the smoothing function.

- For $\sigma>0$ and $x \in R^{d}$, let

$$
\begin{aligned}
\theta_{\sigma}(x) & =\frac{1}{\sigma^{d}} \theta\left(\frac{x}{\sigma}\right) \text { and } \\
\tilde{f}_{\sigma}(x) & =\left\langle f(\cdot), \theta_{\sigma}(x-\cdot)\right\rangle .
\end{aligned}
$$

Definition 1.1. Let $f: R^{d} \rightarrow R$. For a fixed $\sigma>0$, an edge point at scale $\sigma$ along the direction $\mathbf{u}$, where $\mathbf{u}$ is a unit vector in $R^{d}$, is a point $\xi \in R^{d}$ such that the function $\left|\partial_{\mathbf{u}} \tilde{f}_{\sigma}(\xi+t \mathbf{u})\right|^{2}, t \in R$, has a local maximum at $t=0$, i.e., there is a $\delta>0$ such that

$$
\left|\partial_{\mathbf{u}} \tilde{f}_{\sigma}(\xi+t \mathbf{u})\right|^{2}<\left|\partial_{\mathbf{u}} \tilde{f}_{\sigma}(\xi)\right|^{2} \text { whenever }|t| \in(0, \delta) .
$$

Let $E_{\theta}^{(\sigma)}(f ; \mathbf{u})$ be the set of edge points along the direction $\mathbf{u}$ of $f$ at scale $\sigma$. For a point $\xi \in E_{\theta}^{(\sigma)}(f ; \mathbf{u})$ we have

$$
\nabla\left(\left|\partial_{\mathbf{u}} \tilde{f}_{\sigma}(\xi)\right|^{2}\right) \cdot \mathbf{u}=0
$$

Further, let

$$
E_{\theta}^{(\sigma)}(f)=\bigcup_{\mathbf{u}} E_{\theta}^{(\sigma)}(f ; \mathbf{u})
$$

Note that there may be more than one direction along which $f$ has an edge point at $\xi$. Unless otherwise specified, when we say boundaries or contours of $f$, we refer to $E_{\theta}^{(\sigma)}(f)$.

In the literature, an edge point is often defined to be a point $\xi$ where $\left|\nabla \tilde{f}_{\sigma}(x)\right|$ (or equivalently, $\left|\nabla \tilde{f}_{\sigma}(x)\right|^{2}$ ) has a local maximum in the direction of the gradient of $\tilde{f}_{\sigma}$ at $\xi$ (i.e., $\mathbf{u}=\frac{\nabla \tilde{f}_{\tilde{\sigma}}(\xi)}{\left|\nabla \tilde{f}_{\sigma}(\xi)\right|}$ [ [2]. (Note that an edge point in this sense is also an edge point in our sense). In this case, (1.3) changes to

$$
\left(\frac{\nabla \tilde{f}_{\sigma}(\xi)}{\left|\nabla \tilde{f}_{\sigma}(\xi)\right|}\right) \cdot \nabla\left(\left|\nabla \tilde{f}_{\sigma}(\xi)\right|^{2}\right)=0,
$$


which reduces to

$$
\sum_{i j} D_{i j} \tilde{f}_{\sigma}(\xi) D_{i} \tilde{f}_{\sigma}(\xi) D_{j} \tilde{f}_{\sigma}(\xi)=0
$$

This suggests a different and more convenient way of computing the edge points: simply look for the points $\xi$ which satisfy (1.1), although not all these points are edge points in the sense of Definition 1.1 or in the sense mentioned above (before (1.1)). In this connection, in the place of the nonlinear second derivative operator, the Laplacian operator is taken as a convenient approximation (see [2]), and so the points $\xi$ which satisfy

$$
\nabla^{2} \tilde{f}_{\sigma}(\xi)=0
$$

are taken as the edge points. A popular choice for $\theta$ is the Gaussian, $G$, defined as

$$
G(x)=\frac{1}{(2 \pi)^{\left(\frac{d}{2}\right)}} e^{-\frac{|x|^{2}}{2}}, \quad x \in R^{d} .
$$

With $\theta=G$, the points $\xi$ satisfying (1.5) are called the zeros corresponding to the Laplacian of the Gaussian ( $L o G$ ) operator. However, in this work, we shall follow Definition 1.1.

At this point, it is relevant to raise some questions. But before doing so, let us define another class of sets denoted by $\left\{Z_{\theta}^{\sigma}(f), \sigma>0\right\}$. Let an operator, denoted by $\Lambda_{\sigma, \theta}$ (for example, the LoG operator), be given. With this, let

$$
Z_{\theta}^{\sigma}(f)=\left\{\zeta \in R^{d}: \Lambda_{\sigma, \theta} f(\zeta)=0\right\} .
$$

Then the basic questions are

(1) Do the sets $\left\{E_{\theta}^{\sigma}(f), \sigma>0\right\}$ or $\left\{Z_{\theta}^{\sigma}(f), \sigma>0\right\}$ characterize $f$ completely?

(2) If the answer to the above question is negative, then what conditions are to be imposed on $f$ and/or what additional information regarding $f$ is required so as to elicit an affirmative answer?

(3) If the answer is affirmative (possibly after imposing suitable conditions on $f$ and/or invoking additional information), then is there a stable algorithm to retrieve $f$ ?

1.1. Reconstruction of signals or images from partial information. The literature on the representation of signals/images can be classified into four groups, based on the particular formalism used to support the representation: (1) analytic function theory; (2) algebraic geometry; (3) partial differential equations; and (4) others (e.g., wavelet transforms). In this section, we discuss some of these methods in brief. For more details, see [3].

Most of the existing methods center on the representation of signals by zerocrossings, probably inspired by [4] which deals with signals whose real zeros are sufficient to guarantee their uniqueness. According to [4], a bandpass signal of less than one octave having no zeros in common with its Hilbert transform other than real simple zeros is determined by its real zeros (except for a multiplicative constant). The bandpass property is sufficient whereas the other condition is necessary [4]. The necessity of such a stringent condition may be attributed to the restrictiveness arising from a single scale representation. In this scheme, we cannot directly make provision for multiscale representation, since the result applies explicitly to the signal itself. 
A two-dimensional version of Logan's result is formulated in [5], wherein the authors give an iterative algorithm involving the signum and bandpass operators. If their conditions for uniqueness are satisfied, then after convergence we recover the original signal.

The paper by Curtis, Oppenheim, and Lim [6] is a slight departure from our main problem. The authors consider reconstruction of signals from the sign changes of the real part of the Fourier transform of a two-dimensional discrete sequence. The main result of $[6]$ is expressed in terms of the nonfactorizability of the two-variable $z$-transform of the two-variable finite, real sequence.

The paper by Sanz [7, p. 288] aims at finding the conditions for a unique specification of a real polynomial in several variables in terms of their real zeros. The main result states, "Assume that $P$ is a polynomial in $n$ real variables that changes its sign in $R^{n}$. If $P=P_{1}, \ldots, P_{m}$, with all $P_{j}$ distinct and irreducible, then $\operatorname{Sgn}(P)$ determines $P$ uniquely." Sanz [7, pp. 291, 294] concludes that there are two key issues: (i) stability, since the uniqueness results no longer hold when the sign data are sampled; and (ii) discovering effective reconstruction methods.

The paper by Sanz and Huang [8] is similar to [7], except that it concentrates on the two-dimensional version of the problem. The authors claim (see [9]) that the irreducibility condition, apart from being satisfied with probability 1, is stable in the presence of a small perturbation of coefficients. However, note that the stability of irreducibility of polynomials in several variables is different from the stability of the representation using the zeros of the same.

It is to be noted that all the above results are concerned with the conditions for uniqueness or reconstruction of a signal or of an image from its zeros specified at a single scale. In contrast, the paper, for instance, by Hummel and Moniot [10], while exploiting a multiscale representation, examines the appropriateness of the LoG operator and its zeros, keeping in view the requirements of computer vision. The authors attempt to justify the application of the LoG operator by showing that the zero crossings so produced satisfy an evolutionary property. Further, from a knowledge of $\nabla$ LoG $(f)$ at the points of zeros of LoG $(f)$, a blurred version of $\nabla f$ restricted to a finite domain in the scale-space is constructed. The authors treat a digitized version of the problem in the form of a recursive equation, followed ultimately by a formulation in terms of minimization of error.

We now turn our attention to wavelet-based methods. The zero crossings problem in the one-dimensional case is studied in [11]; and the problem involving the maxima of modulus of the gradient in one- and two-dimensional cases is studied in [12]. In [11], the author employs the location of the zeros of the wavelet transform at dyadic scales and the values of the integrals of the wavelet transform with consecutive zeros as the limits of integration. The interpretation is that, when the wavelet is the second derivative of the smoothing function chosen, the integral values are equivalent to the difference between consecutive extrema of the first derivative. Using the method of alternate projections, the author seeks a solution subject to the conditions on (i) the location of the zeros; (ii) the integral values between the successive zeros; and (iii) the resulting solution being a dyadic wavelet transform of a function in $\mathcal{L}^{2}(R)$. In conclusion, the author conjectures that the representation is complete and stable, but acknowledges that this is not so in the subsequent paper [12].

The last reference [12] is very similar to [11] except that the representation is in terms of the local maxima of the modulus of the wavelet transform. A theoretical result is presented to measure the Lipschitz regularity in terms of the decay of the 
wavelet transform when the scale is tending to zero. The reconstruction method, as before, is based on the technique of alternate projections, using which the authors seek a minimum-norm solution. If the locations of maxima points are such that the collection of wavelets with appropriate scale parameters centered on these locations form a frame, then uniqueness of a solution is guaranteed. But, unfortunately, these locations are dependent on the original function itself, and no general sufficient conditions are given.

1.2. An overview of the present work. Suppose that $\mathcal{S}$ is the space of the signals or images, $\mathcal{M}$ the transformed domain corresponding to $\mathcal{S}$, and $T$ the map identifying each member of $\mathcal{S}$ with a member of $\mathcal{M}$. We require $T$ to be one-to-one. Next, we require a stable algorithm to reconstruct $f$ from $T(f)$ for each $f \in \mathcal{S}$.

Let us note some points about $\mathcal{M}$ and $\mathcal{S}$ :

- From a purely computational point of view, we cannot guarantee, in general, that the data points are accurate. However, we can assume a bound on the amount of error in the data, which any general theory of a representation scheme (and of reconstruction therefrom) needs to take into consideration (in a practical problem). This requires that we need to study the problem in an approximate sense (based on the nature of error in the data).

- Our model, $\mathcal{S}$, should reflect reality. That is to say, it is not appropriate to be content with characterizing signals or images that allow a particular representation, though this is a challenging task in mathematical analysis of signals or images, unless the conditions we arrive at are sufficiently general so that all the signals or images that we come across in practice can conveniently be modeled conforming to the characterization. This is because, when we encounter a new signal or image, it may be very difficult (or even impossible) in practice to check whether the specified conditions hold good. But again, the class $\mathcal{S}$ need not be too general.

Bearing these points in mind, we start with a finite dimensional vector space $\mathcal{S}$, and study the approximability of the boundaries with some other set so that the resulting data sets are complete. Specifically, let $N>1$ be a fixed integer, and

$$
\mathbf{L}=\{0,1, \ldots, N-1\}^{d} .
$$

Let $\theta$ be a smoothing function, and

$$
\Theta_{\sigma}(x)=\int_{D} \theta_{\sigma}(x-\xi) d \xi
$$

where $D=\left[0, \frac{1}{N}\right]^{d}$. Then, consider $f$ for which $\tilde{f}_{\sigma}$ has the functional form

$$
\tilde{f}_{\sigma}(x)=\sum_{\mathbf{m} \in \mathbf{L}} C_{\mathbf{m}} \Theta_{\sigma}\left(x-\alpha_{\mathbf{m}}\right),
$$

where $C_{\mathbf{m}} \in R$ and $\alpha_{\mathrm{m}}=\frac{\mathbf{m}}{N}$.

Let $\epsilon>0, \sigma_{1}>\cdots>\sigma_{s}>0$, and $\mathbf{u}_{1}, \ldots, \mathbf{u}_{k}$ be unit vectors in $R^{d}$, where $s$ and $k$ are positive integers. Let $P_{j}\left(f ; \mathbf{u}_{i}\right) \subset R^{d}, 1 \leq j \leq s$, be a finite set satisfying the following property: for every $\zeta \in P_{j}\left(f ; \mathbf{u}_{i}\right)$, there is a $\xi \in E_{\theta}^{\left(\sigma_{j}\right)}\left(f ; \mathbf{u}_{i}\right)$, such that $|\xi-\zeta|<\epsilon . P_{j}\left(f ; \mathbf{u}_{i}\right)$ may be seen as an approximation to a subset of $E_{\theta}^{\left(\sigma_{j}\right)}\left(f ; \mathbf{u}_{i}\right)$. We assume that $P_{j}\left(f ; \mathbf{u}_{i}\right)$ is nonempty when $f$ is nonzero. (This is not a serious assumption, since, as we will see later, for $f \neq 0$ and any $\sigma>0, E_{\theta}^{(\sigma)}(f) \neq \emptyset$.) 
Further, define a map, $\mathcal{A}_{j}$, representing the auxiliary information, as follows:

$$
\mathcal{A}_{j}(\zeta)=\nabla \tilde{f}_{\sigma_{j}}(\zeta), \quad \zeta \in R^{d} .
$$

Suppose now that we are given the sets $M_{j i}(f ; \epsilon), j=1, \ldots, s, i=1, \ldots, k$, where

$$
M_{j i}(f ; \epsilon)=\left\{\left(\zeta, \mathbf{u}_{i} \cdot \mathcal{A}_{j}(\zeta)\right), \quad \zeta \in P_{j}\left(f ; \mathbf{u}_{i}\right)\right\} .
$$

With this, let

$$
\begin{aligned}
M(f ; \epsilon) & =\left(M_{j i}(f ; \epsilon)\right)_{1 \leq j \leq s ; 1 \leq i \leq k} \text { and } \\
\mathcal{M}(\epsilon) & =\{M(f ; \epsilon): f \in \mathcal{S}\} .
\end{aligned}
$$

So all we know about $f$ is the functional form (1.7) and the data sets (1.8), from which we must get the coefficients $C_{\mathbf{m}}$. Now the questions we are interested in are

(1) What are the conditions on $\theta$ under which, for every $\epsilon>0$, there are $\sigma_{j}, \mathbf{u}_{i}$ (independent of $f$ and $\epsilon$ ) and $P_{j}\left(f ; \mathbf{u}_{i}\right), 1 \leq j \leq s, 1 \leq i \leq k$, such that the representation $M(f ; \epsilon)$, as defined in (1.9), uniquely determines $f$ ?

(2) Under those conditions on $\theta$, is the representation $\mathcal{M}(\epsilon)$ in (1.10) stable?

(3) Is there an algorithm to get the coefficients, $C_{\mathbf{m}}$, in a stable manner?

Questions (1) and (2) are central to the next section, where we study the completeness and other theoretical issues. As to question (3), we propose our reconstruction strategy. However, we postpone the details of implementation to section 5. Briefly, our algorithm proceeds in two stages: in the first stage, we get an approximation $h_{j i}$ for $\mathbf{u}_{i} \cdot \mathcal{A}_{j}$; in the second stage, we construct a regularized solution $R(M(f ; \epsilon), h)$, so that even when the data given are in error, we may have the final solution close to the original. While developing the theoretical results, we do not take into account the fact that each point $\zeta \in P_{j}\left(f ; \mathbf{u}_{i}\right)$ is close to a maximum point of $\left|\partial_{\mathbf{u}_{i}} \tilde{f}_{\sigma_{j}}\right|$. We attempt to make up for this by constructing the approximations $h_{j i}$ that preserve shape.

2. Theoretical framework for image representation. We present our models for images and the smoothing function, $\theta$; introduce a new representation scheme; and define the corresponding notions of completeness and stability. After proposing a reconstruction strategy, we demonstrate the existence of a complete representation scheme (with the help of an algorithm to construct one) and its stability. We also present a result on the movement of the edge points with respect to small changes in the images.

2.1. Preliminaries. In the following, $N$ is a fixed positive integer and $\chi_{A}$ is the characteristic function of the set $A$. Consider the set $\mathbf{L}$ defined by

$$
\mathbf{L}=\{0,1, \ldots, N-1\}^{d} .
$$

For $\mathbf{m}=\left(m_{1}, \ldots, m_{d}\right) \in \mathbf{L}$, let

$$
I_{\mathbf{m}}=\left[\frac{m_{1}}{N}, \frac{m_{1}+1}{N}\right) \times \cdots \times\left[\frac{m_{d}}{N}, \frac{m_{d}+1}{N}\right)
$$

and $\alpha_{\mathrm{m}}=\frac{\mathrm{m}}{N}$, i.e.,

$$
\alpha_{\mathrm{m}}=\left(\frac{m_{1}}{N}, \ldots, \frac{m_{d}}{N}\right) .
$$


Let $\mathcal{S}$ be the class of maps $f: R^{d} \rightarrow R$ such that

$$
f(x)=\sum_{\mathbf{m} \in \mathbf{L}} \gamma_{\mathbf{m}} \chi_{I_{\mathbf{m}}}(x) .
$$

Next, we choose the smoothing function $\theta$ to be nonconstant and real-valued, satisfying the following properties:

(1) $\theta \in \mathcal{L}^{1}\left(R^{d}\right)$.

(2) $\theta$ is analytic at every point in $R^{d}$, i.e., at each point $a \in R^{d}$, there is an $r>0$ and a power series representation

$$
\theta(x)=\sum_{k_{1}=0}^{k_{1}=\infty} \ldots \sum_{k_{d}=0}^{k_{d}=\infty} A_{\left(k_{1}, \ldots, k_{d}\right)}\left(x_{1}-a_{1}\right)^{k_{1}} \ldots\left(x_{d}-a_{d}\right)^{k_{d}}
$$

which is convergent for $|x-a|<r$.

(3) $D_{i} \theta \in \mathcal{L}^{1}\left(R^{d}\right)$, and

$$
\lim _{|x| \rightarrow \infty} D_{i} \theta(x)=0
$$

for $1 \leq i \leq d$.

(4) For some $K>0, \quad\left|D_{i j} \theta(x)\right| \leq K \quad \forall x \in R^{d}, 1 \leq i, j \leq d$.

Note that, by (2.1), $D_{i} \theta$ is bounded; hence we can safely assume that $\left|D_{i} \theta\right| \leq K$ and $\left|D_{i j} \theta\right| \leq K$. With this, we infer that $\left|D_{i} \theta_{\sigma}\right| \leq \frac{K}{\sigma^{d+1}}$ and $\left|D_{i j} \theta_{\sigma}\right| \leq \frac{K}{\sigma^{d+2}}$. Also, there is actually a wide range for the choice of $\theta$. Examples include

(1) the Gaussian, $G$, as in (1.6);

(2) $\theta\left(x_{1}, \ldots, x_{d}\right)=\frac{1}{\left(c^{2}+x_{1}^{2}+\cdots+x_{d}^{2}\right)^{m}}$, for $c>0$ and $m>0$ chosen suitably so that $\theta \in \mathcal{L}^{1}\left(R^{d}\right)$

(3) $\theta\left(x_{1}, \ldots, x_{d}\right)=\frac{1}{\left(c_{1}^{2}+x_{1}^{2}\right)^{m_{1}}} \times \cdots \times \frac{1}{\left(c_{d}^{2}+x_{d}^{2}\right)^{m_{d}}}$, for $c_{i}>0$ and $m_{i}>\frac{1}{2}$; and

(4) $\theta\left(x_{1}, \ldots, x_{d}\right)=\frac{e^{-x_{1}}}{\left(1+e^{-x_{1}}\right)^{2}} \times \cdots \times \frac{e^{-x_{d}}}{\left(1+e^{-x_{d}}\right)^{2}}$.

Now for each $f \in \mathcal{S}$,

$$
\begin{aligned}
\tilde{f}_{\sigma}(x) & =\left\langle f(\cdot), \theta_{\sigma}(x-\cdot)\right\rangle \\
& =\int_{R^{d}} \sum_{\mathbf{m} \in \mathbf{L}} \gamma_{\mathbf{m}} \chi_{I_{\mathbf{m}}}(\xi) \theta_{\sigma}(x-\xi) d \xi \\
& =\sum_{\mathbf{m} \in \mathbf{L}} \int_{R^{d}} \gamma_{\mathbf{m}} \chi_{I_{\mathbf{m}}}(\xi) \theta_{\sigma}(x-\xi) d \xi \\
& =\sum_{\mathbf{m} \in \mathbf{L}} \gamma_{\mathbf{m}} \int_{I_{\mathbf{m}}} \theta_{\sigma}(x-\xi) d \xi,
\end{aligned}
$$

where the interchange between the summation and integration in the third step is allowed as the sum is finite. Now the integral in the last step can be written as

$$
\int_{I_{\mathbf{m}}} \theta_{\sigma}(x-\xi) d \xi=\int_{I_{\mathbf{0}}} \theta_{\sigma}\left(x-\alpha_{\mathbf{m}}-\xi\right) d \xi,
$$

where $I_{\mathbf{0}}=\left[0, \frac{1}{N}\right)^{d}$. With $\Theta_{\sigma}(x)=\int_{I_{\mathbf{0}}} \theta_{\sigma}(x-\xi) d \xi$, we get

$$
\tilde{f}_{\sigma}(x)=\sum_{\mathbf{m} \in \mathbf{L}} \gamma_{\mathbf{m}} \Theta_{\sigma}\left(x-\alpha_{\mathbf{m}}\right) .
$$


Now note that $\Theta$ is analytic at each point in $R^{d}$; so also $\tilde{f}_{\sigma}$. Moreover,

$$
D_{i} \Theta_{\sigma}(x)=\int_{I_{0}} \frac{\partial \theta_{\sigma}}{\partial x_{i}}(x-\xi) d \xi
$$

implying that $\left|D_{i} \Theta_{\sigma}\right| \leq \frac{K}{\sigma^{d+1} N^{d}}$, where $K$ is the bound on $\left|D_{i} \theta\right|$. Similarly, it can easily be seen that $\Theta_{\sigma}$ (so also $\tilde{f}_{\sigma}$ ) has bounded derivatives of order $\leq 2$; in fact, $\left|D_{i j} \Theta_{\sigma}\right| \leq \frac{K}{\sigma^{d+2} N^{d}}$. Finally, since $I_{\mathbf{0}}$ is of finite measure, and $D_{i} \theta_{\sigma} \in \mathcal{L}^{1}\left(R^{d}\right)$, by Fubini's theorem, we conclude that $D_{i} \Theta_{\sigma} \in \mathcal{L}^{1}\left(R^{d}\right)$.

2.2. Representation scheme. In this subsection, we define a new representation scheme and formulate the problem of reconstruction of images from their representation.

2.3. Motivation. Suppose we are given information about edge points of $f$ along with the value of the directional derivative at each of these points. We assume that the scales employed are $\sigma_{1}, \ldots, \sigma_{s}, s \geq 1$, and the directions are $\mathbf{u}_{1}, \ldots, \mathbf{u}_{k}, k \geq 1$. Thus we are given $E_{\theta}^{\left(\sigma_{j}\right)}\left(f ; \mathbf{u}_{i}\right), 1 \leq j \leq s, 1 \leq i \leq k$, as well as for each $\xi \in E_{\theta}^{\left(\sigma_{j}\right)}\left(f ; \mathbf{u}_{i}\right)$, $y_{j i}(\xi)=\partial_{\mathbf{u}_{i}} \tilde{f}_{\sigma_{j}}(\xi)$.

Ideally, we would like the information given to us (about $f$ ) to guarantee the uniqueness of the solution, $g$, which at each scale $\sigma_{j}$ and in each direction $\mathbf{u}_{i}$ satisfies the following conditions:

(1) At each point $\xi \in E_{\theta}^{\left(\sigma_{j}\right)}\left(f ; \mathbf{u}_{i}\right)$, we have $\partial_{\mathbf{u}_{i}} \tilde{g}_{\sigma_{j}}(\xi)=y_{j i}(\xi)$.

(2) Each point $\xi \in E_{\theta}^{\left(\sigma_{j}\right)}\left(f ; \mathbf{u}_{i}\right)$ is an edge point of $g$ along the direction $\mathbf{u}_{i}$.

(3) No point $\xi \notin E_{\theta}^{\left(\sigma_{j}\right)}\left(f ; \mathbf{u}_{i}\right)$ is an edge point of $g$ along $\mathbf{u}_{i}$.

In the case of images, $E_{\theta}^{(\sigma)}(f ; \mathbf{u})$ is infinite whenever it is nonempty (see the next section). However, we assume that we are given only finitely many points for each $\sigma=\sigma_{j}$; hence, we may do away with condition (3) in this case.

Observe that only condition (1) can be expressed in the form of equations. Because of the difficulty in dealing with condition (2) (as also with condition (3)) above, we need to modify our representation so that we use only condition (1) in order to establish uniqueness and other theoretical results. Further, in the reconstruction algorithms, we try to account for the geometrical significance of the data by developing certain heuristics to guide the solution.

Proposed representation. In the following, let $\epsilon$ be a fixed positive real number. For a nonzero $g \in \mathcal{S}$, let $P_{j i}(g)$ be a finite set such that, for each $\zeta \in P_{j i}(g)$, there is a $\xi \in E_{\theta}^{\left(\sigma_{j}\right)}\left(g ; \mathbf{u}_{i}\right)$ such that $|\xi-\zeta|<\epsilon$. Define

$$
M_{j i}(g)=\left\{\left(x, \mathcal{A}_{i} \tilde{g}_{\sigma_{j}}(x)\right): x \in P_{j i}(g)\right\},
$$

where $\mathcal{A}_{i} \tilde{g}_{\sigma_{j}}=\partial_{\mathbf{u}_{i}} \tilde{g}_{\sigma_{j}}$. Finally, let

$$
\mathcal{M}=\{M(g): g \in \mathcal{S}\}
$$

where $M(g)$ is the $s \times k$ "matrix" whose elements are $M_{j i}(g)$. We assume that $\rho_{\mathcal{M}}$ and $\rho_{\mathcal{S}}$ are the metrics on $\mathcal{M}$ and $\mathcal{S}$, respectively. With this, we introduce the following terminology.

DEFINITION 2.1. $M(g)$, called a representation of $g$, is a complete representation of $g$ if for any $h \in \mathcal{S}$, 


$$
\partial_{\mathbf{u}_{i}} \tilde{h}_{\sigma_{j}}(x)=y, \quad(x, y) \in M_{j i}(g)
$$

for $1 \leq i \leq k$ and $1 \leq j \leq s$, then $h=g$.

DEFINITION 2.2. $\mathcal{M}$, called a representation scheme, is said to be complete if $M(g)$ is complete for every $g \in \mathcal{S} \backslash\{0\} . \mathcal{M}$ is said to be stable if, for every $g \in \mathcal{S} \backslash\{0\}$, there are $a \delta_{0}>0$ and a continuous real-valued function $\eta(t), t \in\left[0, \delta_{0}\right)$, with $\eta(0)=0$, such that $\forall \delta$ with $0<\delta<\delta_{0}$,

$$
\rho_{\mathcal{M}}(M(g), M(h))<\delta \Longrightarrow \rho_{\mathcal{S}}(g, h)<\eta(\delta),
$$

holds whenever $h \in \mathcal{S} \backslash\{0\}$.

\subsection{Observations.}

(1) If $M(g)$ is complete, $h \in \mathcal{S}$, and $M(g)=M(h)$, then $g=h$.

(2) Note that the identically 0 function does not have any edge points; hence $M(0)=\emptyset$. Therefore, in the definition of a complete representation scheme, we require that $M(g)$ be complete only for $g \neq 0$.

(3) Let $T: \mathcal{S} \rightarrow \mathcal{M}$ be the map sending $f$ to $M(f)$. If $\mathcal{M}$ is complete, then $T$ is one-to-one.

(4) Stability of $\mathcal{M}$ implies that $T$ is one-to-one. To see this, note that if $T$ is not one-to-one, then we can find $f, g \in \mathcal{S}$ such that $f \neq g$, but $M(f)=M(g)$; thus for every $\delta>0$, we have $\rho_{\mathcal{M}}(M(g), M(h))=0<\delta$; but, since $\eta$ is continuous at 0 and $\eta(0)=0$, there is a $\delta_{0}$ such that $\eta(\delta)<\rho_{\mathcal{S}}(f, g)$, whenever $\delta<\delta_{0}$; hence $\mathcal{M}$ is not stable.

(5) The condition for $\mathcal{M}$ to be stable may be rewritten as the requirement that the map $T^{-1}$ (which is well defined because $T$ is one-to-one, and $\mathcal{M}$ is precisely the image of $\mathcal{S}$ under $T$ ), sending $M(f)$ to $f$, be continuous at every $f \neq 0$.

Later, we shall show that when we choose $\mathbf{u}_{i}$ to be the standard basis vectors for $R^{d}$, a complete representation scheme can always be constructed for every $\epsilon>0$, and that a representation scheme is stable whenever it is complete.

Formulation of the reconstruction problem. To begin with, we do not know whether the information is sufficient to guarantee uniqueness. Hence, we should formulate the reconstruction problem independent of any such assumptions; also, we should make provision in our formulation for obtaining the exact solution in case such a result holds. It would always be an advantage to have the formulation and reconstruction mechanism flexible in terms of the amount of inputs required. Keeping these things in mind, we propose our reconstruction strategy as an instance of the regularization method. However, the details of implementation are postponed to section 6, where we present experimental results in which the number of data points is much smaller than the number of unknowns; more precisely we reconstruct images from edge points along the standard basis vectors at just a single scale.

For each $g \in \mathcal{S}$ and $M \in \mathcal{M}$, let

$$
\mu(g, M)=\sum_{j=1}^{j=s} \sum_{i=1}^{i=k} \sum_{(x, y) \in M_{j i}}\left|\mathcal{A}_{i} \tilde{g}_{\sigma_{j}}(x)-y\right|^{2} .
$$

Further, let a parametric family of nonnegative real-valued continuous functions, $\omega_{\nu}, \nu \in J\left(=[0, \infty)^{k}\right)$, be defined on $\mathcal{S} \times \mathcal{M}$, which satisfies, for each $M \in \mathcal{M}$, the following properties:

(1) The functional $\omega_{\nu}(\cdot, M)$-called the shaper functional — defined on $\mathcal{S}$ obtained by restricting $\omega_{\nu}$ to $\mathcal{S} \times\{M\}$ is strictly convex. 
(2) For each $\eta>0$, the set $A=\left\{g \in S: \omega_{\nu}(g, M)<\eta\right\}$ is a bounded subset of $\mathcal{S}$.

(3) $\lim _{\nu \rightarrow 0} \omega_{\nu}(g, M)=\mu(g, M)$ for $g \in S$.

We use $\omega_{\nu}$ as a measure of the departure of the reconstructed image from the original in terms of shape: for a fixed $M$, the smaller the value of $\omega_{\nu}(g, M)$, the better the shape of $g$ (hence the name shaper functional). We shall construct it in section 6 . With this, we formulate our reconstruction problem as follows.

Reconstruction from modulus maxima (RMM): Given an $M \in \mathcal{M}$, find an image $g_{\nu}=\mathcal{R}_{\nu}(M) \in \mathcal{S}$, such that $\omega_{\nu}\left(g_{\nu}, M\right)$ is minimum.

There are several points to be noted. In the first place, we are guaranteed a unique reconstruction whenever $M$ is nonempty. Also, the reconstructed solution $\mathcal{R}_{\nu}(M)$ is continuous with respect to $M$. Finally, if $M=M(f)$ is complete, then as $c \rightarrow 0$, $\mathcal{R}_{\nu} \rightarrow f$. For details on the regularization method, see [13].

An important aspect of the above formulation is the cost functional $\omega_{\nu}(\cdot, M)$, since, in practice, we do not actually set $\nu=0$ to guard against the possible illconditionality of the resulting system of equations. In fact, if we use an incomplete representation, then the system becomes singular (when $\nu=0$ ). Also, the formulation does not directly take into account the fact that the data are near the edge points of the original; the only link between this fact and the formulation of the reconstruction problem is this functional. For these reasons, this functional is our main interest in the section on experimental results where we show reconstructions using this (but with an incomplete representation).

At this point, it is of interest to know what happens to the boundaries of the regularized solution as $\nu \rightarrow 0$. Suppose $M=M(f)$ and $M$ is complete. Then, we have these questions:

(1) For every edge point $x$ of $f$, is there a sequence $\left\{x_{\nu}\right\}$ of edge points of $\mathcal{R}_{\nu}(M)$ converging to those of $f$ as $\nu \rightarrow 0$ ?

(2) Do the sets $E_{\theta}^{(\sigma)}\left(\mathcal{R}_{\nu}(M) ; \mathbf{u}\right)$ converge to $E_{\theta}^{(\sigma)}(f ; \mathbf{u})$ as $\nu \rightarrow 0$ with respect to any "reasonable" distance measure?

We shall see below (in section 5) that the answer to the first question is yes. The second question is not answered in this work.

In the following sections, we present the results showing the existence of a complete representation and its stability. In the process, we deal with the problem of reconstructing $f$ from the modulus maxima of the convolved versions of $f$. The applicability of these results to our problem is discussed later.

3. Existence of a complete representation scheme. In this section, we discuss the uniqueness of the solution $f$ when information about maxima points of $\left|\tilde{f}_{\sigma}\right|$ is given. We assume that the convolving function is $\psi$, satisfying the following properties: $\psi \in \mathcal{L}^{1}\left(R^{d}\right), \psi \neq 0, \psi$ is analytic at every point of $R^{d}$, and $\lim _{|x| \rightarrow \infty} \psi(x)=0$.

We denote the maxima points of $\left|\tilde{f}_{\sigma}\right|$ with the symbol $E_{\psi}^{(\sigma)}(f)$. By a maximum point we mean a point $\xi \in R^{d}$ such that the function $\left|\tilde{f}_{\sigma}(\xi+t \mathbf{u})\right|, t \in R$, has a maximum at $t=0$ for some $\mathbf{u}$. Let $\Psi_{\sigma}$ be defined by $\Psi_{\sigma}(x)=\int_{I_{0}} \psi_{\sigma}(x-\xi) d \xi$, where $I_{\mathbf{0}}$ is as defined in the previous section. Specifically, we address the following question: Given $f \neq 0$, is there a positive integer $s$ such that for any $\sigma_{1},>\cdots>$ $\sigma_{s}>0$, we can find $P_{j}(f), 1 \leq j \leq s, 1 \leq i \leq d$, with Properties 1 and 2 listed below?

(1) For each $x \in P_{j}(f)$, there is a $\xi \in E_{\psi}^{\left(\sigma_{j}\right)}(f)$ with $|x-\xi|<\epsilon$.

(2) If for any $g \in \mathcal{S}, \tilde{g}_{\sigma_{j}}(x)=\tilde{f}_{\sigma_{j}}(x), \quad x \in P_{j}(f)$, then $g=f$. 
We shall show the applicability of these results to our problems later. We begin our study with a few elementary properties of the set $\mathcal{S}$. Let $\rho_{\mathcal{S}}$ be the sup-metric on $\mathcal{S}$, i.e., $\rho_{\mathcal{S}}(f, g)=\max _{\mathbf{m} \in \mathbf{L}}\left\{\left|\gamma_{\mathbf{m}}-\lambda_{\mathbf{m}}\right|\right\}$, where

$$
f=\sum_{\mathbf{m} \in \mathbf{L}} \gamma_{\mathbf{m}} \chi_{I_{\mathbf{m}}} \text { and } g=\sum_{\mathbf{m} \in \mathbf{L}} \lambda_{\mathbf{m}} \chi_{I_{\mathbf{m}}} .
$$

Write $|f|_{\mathcal{S}}=\rho_{\mathcal{S}}(f, 0)$. It is easy to see that $|\cdot|_{\mathcal{S}}$ is a norm on $\mathcal{S}$ and that with this norm $\mathcal{S}$ becomes a Banach space. Finally let

(1) $\dot{\mathcal{S}}=\mathcal{S} \backslash\{0\}$,

(2) $\mathcal{S}^{1}=\left\{h \in \mathcal{S}:|h|_{\mathcal{S}} \leq 1\right\}$, and

(3) $\mathcal{D}^{1}=\left\{h \in \mathcal{S}:|h|_{\mathcal{S}}=1\right\}$.

With this notation, we start with the following proposition.

Proposition 3.1. Let $f \in \mathcal{S} . \tilde{f}_{\sigma} \equiv 0 \Longrightarrow f \equiv 0$.

Proof. Write $F=\tilde{f}_{\sigma}$. Now, by definition

$$
F(x)=\left\langle f(\cdot), \psi_{\sigma}(x-\cdot)\right\rangle .
$$

By our discussion in section 2.1, we have $F \in \mathcal{L}^{1}\left(R^{d}\right)$. Taking the Fourier transform on both sides of (3.1), we get $\hat{F}=\hat{f} \hat{\psi}_{\sigma}$. Suppose that $F \equiv 0$. Since $\psi_{\sigma} \not \equiv 0$ and is continuous, $\hat{\psi}_{\sigma} \not \equiv 0$. So there is a point $\omega \in R^{d}$ such that $\hat{\psi}(\omega) \neq 0$. But since $\psi_{\sigma} \in$ $\mathcal{L}^{1}\left(R^{d}\right), \hat{\psi}_{\sigma}$ is continuous. So there is an open ball, $B(\omega ; \delta)$ with $\delta>0$, throughout which $\psi_{\sigma}$ does not vanish. Now since $\hat{F} \equiv 0$, we must have $\hat{f}(y)=0, y \in B(\omega ; \delta)$. Also, since $f$ is compactly supported, by the Paley-Wiener theorem, $\hat{f}$ is entire. Now if $x \in R^{d} \backslash B(\omega ; \delta)$, with $\mathbf{u}=\frac{x-\omega}{|x-\omega|}$, consider the function $h(z)=\hat{f}(\omega+z \mathbf{u}), z \in C$. Now $h$ is an entire function of one complex variable. But since $\hat{f}(y)=0, y \in B(\omega ; \delta)$, $h(t)=0$ for $t \in(0, \delta)$, implying that $h \equiv 0$. Now if we take $t=|x-\omega|$, then $h(t)=\hat{f}(x)$. Thus $\hat{f}(x)=0$. But $x$ is an arbitrary point in $R^{d} \backslash B(\omega ; \delta)$. So $\hat{f}$ is zero throughout $R^{d}$. Hence $f=0$ almost everywhere on $R^{d}$. Now consider the functional form of $f: f=\sum_{\mathbf{m}} \gamma_{\mathbf{m}} \chi_{I_{\mathbf{m}}}$. It is easy to see that $\gamma_{\mathbf{m}}=0$, since $I_{\mathbf{m}}$ has positive measure and is disjoint from $I_{\mathbf{k}}$ for $\mathbf{m} \neq \mathbf{k}$.

Proposition 3.2. If $f \in \dot{\mathcal{S}}$, then for any $x \in R^{d}$, $\left|\tilde{f}_{\sigma}\right|$ is not constant in any neighborhood of $x$.

Proof. Suppose for some $\delta>0,\left|\tilde{f}_{\sigma}\right|$ is constant throughout $B(x ; \delta)$. Take any unit vector $\mathbf{u}$ and consider the function

$$
\phi(t)=\left|\tilde{f}_{\sigma}(x+t \mathbf{u})\right|^{2}, \quad t \in R .
$$

Then $\phi$ is constant in $(0, \delta)$. Since $\phi$ is analytic at every point in $R$, we conclude that $\phi$ is constant throughout. If now $y \in R^{d} \backslash B(x ; \delta)$, then taking $\mathbf{u}=\frac{y-x}{|y-x|}$ and $t=$ $|y-x|$, we see that $\left|\tilde{f}_{\sigma}(y)\right|^{2}=\left|D_{i} \tilde{f}_{\sigma}(x)\right|^{2}$. But since $y$ is arbitrary, we conclude that $\left|\tilde{f}_{\sigma}\right|^{2}$ is constant. However, since $\lim _{|x| \rightarrow \infty}|\psi(x)|=0$, so also $\lim _{|x| \rightarrow \infty}\left|\tilde{f}_{\sigma}(x)\right|=0$, implying that $\left|\tilde{f}_{\sigma}\right| \equiv 0$; and hence $f \equiv 0$, contrary to the hypothesis.

Proposition 3.3. If $f \in \dot{\mathcal{S}}$, then $E_{\psi}^{(\sigma)}(f)$ is nonempty.

Proof. Since $f \neq 0,\left|\tilde{f}_{\sigma}\right| \not \equiv 0$ by Proposition (3.1). Also, since $\lim _{|x| \rightarrow \infty}\left|\tilde{f}_{\sigma}(x)\right|=$ 0 , there is a global maximum of $\left|\tilde{f}_{\sigma}\right|$ at some point $\xi$. Note that $\tilde{f}_{\sigma}(\xi) \neq 0$. Now consider the function $\phi$ defined by

$$
\phi(t)=\left|\tilde{f}_{\sigma}(\xi+t \mathbf{u})\right|^{2}, \quad t \in R,
$$


for some unit vector $\mathbf{u}$. We claim that for some $\delta>0, \phi$ satisfies

$$
\phi(0)>\phi(t), \quad 0<|t|<\delta .
$$

Suppose (3.3) holds for no $\delta$. Then, for each positive integer $n$ with $\delta_{n}=\frac{1}{n}$, there is a point $t_{n}$ in $\left(0, \delta_{n}\right)$ such that $\phi(0) \leq \phi\left(t_{n}\right)$. However, since at $\xi,\left|\tilde{f}_{\sigma}\right|$ achieves its global maximum, $\phi(0)$ is the global maximum for $\phi$; so $\phi(0) \geq \phi\left(t_{t_{n}}\right)$. Combining with the earlier inequality, we get $\phi(0)=\phi\left(t_{n}\right)$. From this, we get an infinite sequence $h_{k}=t_{n_{k}}$, such that $0<h_{k+1}<h_{k}$, and $\phi\left(h_{k}\right)=\phi(0)$. Now since $\phi$ is analytic, and the sequence $\left\{h_{k}\right\}$ converges, $\phi$ is identically equal to a constant. But since $\lim _{t \rightarrow \infty} \phi(t)=0$, this constant must be 0 ; so, in particular, $\phi(0)=\tilde{f}_{\sigma}(\xi)=0$, contrary to the choice of $\xi$. Hence, there is no such sequence $\left\{t_{n}\right\}$, which implies that there is a $\delta_{1}>0$ such that $\phi(t)<\phi(0), t \in\left(0, \delta_{1}\right)$. Similarly, we get a $\delta_{2}>0$ such that $\phi(t)<\phi(0), t \in\left(-\delta_{2}, 0\right)$. Now let $\delta=\min \left\{\delta_{1}, \delta_{2}\right\}$. It is easy to see that for $|t| \in(0, \delta), \phi(t)<\phi(0)$, thereby implying that $\xi \in E_{\psi}^{\sigma}(f)$.

TheOREm 3.4 (completeness). Let $\epsilon>0, f \in \dot{\mathcal{S}}$. Then, for any distinct $\sigma_{j}>$ $0,1 \leq j \leq N^{d}$, we can find finite sets $P_{j}(f)$ satisfying the following conditions:

(1) For each $x \in P_{j}(f)$, there is a $\xi \in E_{\psi}^{\left(\sigma_{j}\right)}(f)$ with $|x-\xi|<\epsilon$.

(2) The system

$$
\sum_{\mathbf{m} \in \mathbf{L}} \gamma_{\mathbf{m}} \Psi_{\sigma_{j}}\left(x-\alpha_{\mathbf{m}}\right)=\tilde{f}_{\sigma_{j}}(x), \quad x \in P_{j}(f), 1 \leq j \leq s
$$

can be solved uniquely for $\gamma_{\mathrm{m}}$.

Proof. We give a constructive proof with the help of an algorithm for obtaining a complete representation. Set $n=1$, and let $\xi_{n} \in E_{\psi}^{\left(\sigma_{n}\right)}(f)$. Set $P_{n}=\emptyset$. Construct a point evaluation functional, $T_{n} \in \mathcal{S}^{\star}$, using the following iterative scheme:

(1) If $n>N^{d}$, then stop.

(2) If $n=1$, then pick any $g_{n} \in \mathcal{D}^{1}$. Otherwise $(n>1)$, choose $g_{n} \in \mathcal{D}^{1}$ such that $T_{j}\left(g_{n}\right)=0,1 \leq j \leq n-1$.

By Proposition 3.2, $\tilde{g}_{\sigma_{n}}$ is nonconstant in $B\left(\xi_{n}, \epsilon\right)$. Hence $\exists x_{n} \in B\left(\xi_{n}, \epsilon\right)$ such that $\tilde{g_{n}} \sigma_{n}\left(x_{n}\right) \neq 0$. Set $P_{n}=P_{n} \bigcup\left\{x_{n}\right\}$.

(3) With $\xi_{n}$ as in the previous step, choose $T_{n}$ to be the linear functional that sends each $h \in \mathcal{S}$ to $\tilde{h}_{\sigma_{n}}\left(x_{n}\right)$.

(4) Choose $x_{n+1}$ to be a point in $E_{\psi}^{\left(\sigma_{n+1}\right)}(f)$, and $P_{n+1}$ to be any set satisfying property 1 (for example, $\emptyset$ ).

(5) Set $n \leftarrow n+1$, and go to step 1.

Claim: The sets $P_{n}$ have the required properties.

Observation: We need to check only property 2 .

Suppose property 2 does not hold. Then there is an $h \in \mathcal{D}^{1}$ such that

$$
\tilde{h}_{\sigma_{n}}\left(x_{n}\right)=0 \forall n \Longrightarrow T_{n}(h)=0 \forall n .
$$

But note that $T_{1}, \ldots, T_{n}$ are linearly independent, since $T_{n}\left(g_{n}\right)=\tilde{g_{n_{\sigma_{n}}}}\left(x_{n}\right) \neq 0$ but $T_{j}\left(g_{n}\right)=0,1 \leq j \leq n-1$ (see steps 2 and 3 of the construction). Hence, $\left\{T_{1}, \ldots, T_{N^{d}}\right\}$ is a basis for $\mathcal{S}^{\star}$. Therefore, (3.4) implies that $h=0$, contrary to our assumption that $h \in \mathcal{D}^{1}$.

It is important to note that in the previous theorem, we are completely free to choose $\sigma_{j}$ 's as we please. The requirement of employing more than one scale may be relaxed when $d \geq 2$ because of the following proposition. 
Proposition 3.5. If $d \geq 2$ and $f \in \dot{\mathcal{S}}$, then $E_{\psi}^{(\sigma)}(f)$ is infinite.

Proof. Let $\xi \in E_{\psi}^{(\sigma)}(f)$. Then, there are $\mathbf{u}$ and $\delta$ such that the function $\phi$ as defined in (3.2) satisfies $\phi(t)<\phi(0)$ for $|t| \in(0, \delta)$. It follows that

$$
\phi^{\prime}(t)>0 \text { for } t \in(-\delta, 0) \text { and } \phi^{\prime}(t)<0 \text { for } t \in(0, \delta) .
$$

Now, consider the function $F: R^{d+1} \rightarrow R$ defined by

$$
F(x, t)=\left|\tilde{f}_{\sigma}(x+t \mathbf{u})\right|^{2},
$$

so that $\phi(t)=F(\xi, t)$, and $\phi^{\prime}(t)=\frac{\partial F(\xi, t)}{\partial t}$. Also, by $(3.5), \phi^{\prime}(-a)>0$ and $\phi^{\prime}(a)<0$, where $a=\frac{\delta}{2}$. Now since $F$ is continuously differentiable, there is an $\eta>0$, such that (i) $\frac{\partial F(x, t)}{\partial t}>0$, whenever $|x-\xi|<\eta$ and $|t+a|<\eta$, and (ii) $\frac{\partial F(x, t)}{\partial t}<0$, whenever $|x-\xi|<\eta$ and $|t-a|<\eta$. Therefore, for each $x \in B(\xi, \eta), \frac{\partial F(x, t)}{\partial t}$ changes its sign along the line $L_{x}(t)=x+\mathbf{u} t$. It is easy to see that there is a maximum point, $\zeta_{x}$, for $F(x, t)$ along $L_{x}$. Recalling the definition of $F(x, t)$ from (3.6), we conclude that $\zeta_{x} \in E_{\psi}^{(\sigma)}(f)$.

In view of the previous proposition, with a minor modification of the proof given for Theorem 3.4, we can construct $P(f)$ corresponding to a single scale $\sigma$ with an adequate number of points in it. Let us state it formally.

Theorem 3.6. Let $\sigma, \epsilon>0, f \in \dot{\mathcal{S}}$. Then, there is a finite set $P(f)$ satisfying the following properties:

(1) For each $x \in P(f)$, there is $a \xi \in E_{\psi}^{(\sigma)}(f)$ with $|x-\xi|<\epsilon$.

(2) The system

$$
\sum_{\mathbf{m} \in \mathbf{L}} \gamma_{\mathbf{m}} \Psi_{\sigma}\left(x-\alpha_{\mathbf{m}}\right)=\tilde{f}_{\sigma}(x), \quad x \in P(f), 1 \leq j \leq s,
$$

can be solved uniquely for $\gamma_{\mathbf{m}}$.

Proof. We just present the algorithm to construct $P(f)$. The proof for the fact that $P(f)$ has the required properties is entirely analogous to the one given for Theorem 3.4.

Set $n=1$, and let $\xi_{n} \in E_{\psi}^{(\sigma)}(f)$. Choose $P(f)$ to be any set (for example, $\emptyset$ ) satisfying property 1 . Set $n=1$, and construct a point evaluation functional, $T_{n} \in \mathcal{S}^{\star}$, using the following iterative scheme:

(1) If $n>N^{d}$, then stop.

(2) If $n=1$, then pick any $g_{n} \in \mathcal{D}^{1}$. Otherwise $(n>1)$, choose $g_{n} \in \mathcal{D}^{1}$ such that $T_{j}\left(g_{n}\right)=0,1 \leq j \leq n-1$. By Proposition $3.2, \tilde{g_{n_{\sigma_{n}}}}$ is nonconstant in $B\left(x_{n}, \epsilon\right)$. Hence $\exists x_{n} \in B\left(\xi_{n}, \epsilon\right)$ such that $\tilde{g_{n}} \sigma_{n}\left(x_{n}\right) \neq 0$. Set $P=P \bigcup\left\{x_{n}\right\}$.

(3) With $x_{n}$ as in the previous step, choose $T_{n}$ to be the linear functional that sends each $h \in \mathcal{S}$ to $\tilde{h}_{\sigma_{n}}\left(x_{n}\right)$.

(4) Choose $\xi_{n+1}$ to be a point in $E_{\psi}^{(\sigma)}(f) \backslash\left\{\xi_{1}, \ldots, \xi_{n}\right\}$.

(5) Set $n \leftarrow n+1$, and go to step 1 .

What the previous theorems say is that, for each nonzero $f \in \mathcal{S}$, to an arbitrary precision we can always "approximate" the edge-point set, $E_{\psi}^{\left(\sigma_{j}\right)}(f)$, with a finite set $P_{j}(f)$ in such a way that the map $T$ sending $f$ to $M(f)$, where $M(f)=$ $\left(M_{1}(f), \ldots, M_{s}(f)\right)$, and $M_{j}(f)=\left\{\left(x, \tilde{f}_{\sigma_{j}}(x)\right): x \in P_{j}(f)\right\}$, is one-to-one. Note that it is enough to construct $P_{j}(f)$ for $|f|_{\mathcal{S}}=1$ since we can choose $P_{j}(c f)=P_{j}(f)$ for any $c \neq 0$. Let $\mathcal{M}=\{M(f): f \in \mathcal{S}\}$. We know that the map $T$ sending $f$ 
to $M(f)$ is one-to-one for $f \neq 0$. A natural way to define a metric $\rho_{\mathcal{M}}$ on $\mathcal{M}$ is to take the Hausdorff metric on each of the component sets $M_{j i}$ considered as subsets of $R^{d+1}$, and take $\rho_{\mathcal{M}}$ to be their sum. Define $\dot{\mathcal{M}}=\{M(f): f \in \dot{\mathcal{S}}\}$. We have $\dot{\mathcal{S}}$ with $\rho_{\mathcal{S}}$ restricted to it on the one hand and $\dot{\mathcal{M}}$ with $\rho_{\mathcal{M}}$ restricted to it on the other. The question is, How are $\rho_{\mathcal{S}}$ and $\rho_{\mathcal{M}}$ related? We shall prove in the next section that $T^{-1}$ restricted to $\dot{\mathcal{M}}$ is locally Lipschitz; hence, for every $M(f) \in \dot{\mathcal{M}}$, there are constants $\delta_{0}, K_{0}>0$, depending on $M(f)$, such that $\rho_{\mathcal{S}}(f, g) \leq K_{0} \cdot \rho_{\mathcal{M}}(M(f), M(g))$ whenever $\rho_{\mathcal{M}}(M(f), M(g))<\delta_{0}$. We shall also show, in the last section, that whenever $g_{n} \rightarrow f$, then for every edge point $x$ of $f$, there is a sequence $\left\{x_{n}\right\}$ where $x_{n}$ is an edge point of $g_{n}$ such that $x_{n} \rightarrow x$.

Before closing this section, we shall show the applicability of the above results to our original problems. Note that since $\theta$ is nonconstant, there is an $i$ such that $D_{i} \theta \neq 0$. In fact, this holds for every $i$, since $\theta \in \mathcal{L}^{1}\left(R^{d}\right)$. With $\psi=D_{i} \theta$, it can be seen that Propositions 3.1 and 3.2 hold without any modification. In the proof of Proposition 3.3, we have to choose $\mathbf{e}_{i}$ in place of $\mathbf{u}$, where $\mathbf{e}_{i}$ is the $i$ th standard basis vector. Theorems 3.4 and 3.6 hold without modification as they depend only on the previous propositions. With this, we move to the next section where we prove that any complete representation scheme is stable.

4. Stability of a complete representation scheme. In this section, we show that any complete representation scheme is stable. So let $\mathcal{M}$ be a representation scheme. Fix an $M=M(f) \in \mathcal{M}$. Recall our construction of $M$ : we have $M=\left(M_{j i}\right)$, where $M_{j i}=\left\{\left(x, \partial_{\mathbf{u}_{i}} \tilde{f}_{\sigma_{j}}\right): x \in P_{j i}(f)\right\}$, and $P_{j i}(f)$ is such that for every $x \in P_{j i}(f)$, there is a $\xi \in E_{\theta}^{\left(\sigma_{j}\right)}\left(f ; \mathbf{u}_{i}\right)$ with $|x-\xi|<\epsilon$. Also, by the completeness of $\mathcal{M}$, the linear system of linear equations

$$
\sum_{\mathbf{m} \in \mathbf{L}} \lambda_{\mathbf{m}} \partial_{\mathbf{u}_{i}} \Theta_{\sigma_{j}}\left(x-\alpha_{\mathbf{m}}\right)=y, \quad(x, y) \in M_{j i}
$$

is consistent and can be uniquely solved for $\lambda_{\mathbf{m}}$. Note that $\mathbf{u}_{i}$ and $\sigma_{j}$ are independent of $f$. We assume that $\mathcal{M}$ is equipped with the metric $\rho_{\mathcal{M}}$ defined as $\rho_{\mathcal{M}}(M(f), M(g))=$ $\sum_{j, i} \rho_{\mathcal{H}}\left(M_{j i}(f), M_{j i}(g)\right)$, with $\rho_{\mathcal{H}}$, the Hausdorff metric on the nonempty compact subsets of $R^{d+1}$, given by $\rho_{\mathcal{H}}(A, B)=\max \{D(A, B), D(B, A)\}$, where

$$
D(X, Y)=\max _{x \in X}\left\{\min _{y \in Y}\{|x-y|\}\right\} \text {. }
$$

Our objective here is to show that for every $f \in \dot{\mathcal{S}}$ and any sequence $\left\{f_{n}\right\} \subset \mathcal{S}$, as $M\left(f_{n}\right) \rightarrow M(f), f_{n} \rightarrow f$. In fact, under the given conditions, we show that, for some $\delta_{0}, K_{0}>0$, depending on $M(f)$, whenever $\rho_{\mathcal{M}}(M(g), M(f))<\delta_{0}$, we have $|g-f|_{\mathcal{S}} \leq K_{0} \cdot \rho_{\mathcal{M}}(M(g), M(f))$. This proves, in particular, that the map $T^{-1}$ sending $M(f)$ to $f$, when restricted to $\dot{\mathcal{M}}=\{M(g): g \in \dot{\mathcal{S}}\}$, is locally Lipschitz. We shall start with a short study of the behavior of the linear system (4.1).

Uniqueness of the solution for $\lambda_{\mathrm{m}}$ in (4.1) implies that there are $N^{d}$ linearly independent equations that can be extracted from the same system. Choose one such system (arbitrarily), and construct an $N^{d} \times N^{d}$ matrix $A_{M}$ whose elements are the coefficients of the $\lambda_{\mathrm{m}}$ in the chosen system of linearly independent equations. Index the elements by $\mathbf{m n}$, where $\mathbf{m}, \mathbf{n} \in \mathbf{L}$. Similarly, construct a $1 \times N^{d}$ matrix $C_{M}$ whose entries are the right-hand-side members of the independent system of equations chosen (taken in the same order as the rows of $A$ ). Note that the elements of $C_{M}$ are just 
real numbers, whereas those of $A_{M}$ are the values of certain functions evaluated at certain points (in $R^{d}$ ). As we move in the space $\mathcal{M}$, say from $M$ to $M^{\prime}$, the variables involved are the elements of $C_{M}$, as are the points of evaluation of the functions that appear in $A_{M}$; hence the change in $A_{M}$ is a little more complicated than that in $C_{M}$. More importantly, because of the arbitrariness of our choice of $A_{M}$, we cannot expect $A_{M^{\prime}}$ to be close to $A_{M}$ when $M^{\prime}$ is close to $M$, unless care is taken in the choice of $A_{M^{\prime}}$; the same applies to $C_{M}$ and $C_{M^{\prime}}$, as well. We discuss these issues later. For now, we shall concentrate on the study of the properties of $A_{M}$ viewed as a matrix of functions. To do this, let us introduce some notation. The applicability of the following results to the present case is postponed until the end of the section.

Let $\mathcal{T}$ be the space of all $N^{d} \times N^{d}$ matrices, $A$, whose $\mathbf{m n t h}$ element, $a_{\mathbf{m n}},{ }^{1}$ has the form

$$
a_{\mathbf{m n}}=a_{\mathbf{m n}}\left(x_{\mathbf{m}}\right)=D_{\mathbf{m}} \Theta_{\tau_{\mathbf{m}}}\left(x_{\mathbf{m}}-\alpha_{\mathbf{n}}\right),
$$

where $x_{\mathbf{m}} \in R^{d}, \tau_{\mathbf{m}}=\sigma_{j(\mathbf{m})}$, and $D_{\mathbf{m}}=\partial_{\mathbf{u}_{i(\mathbf{m})}}$. Note that $D_{\mathbf{m}}$ are all fixed but not necessarily different; the same is true of $\tau_{\mathbf{m}}$. But $x_{\mathbf{m}}$ are treated as independent variables; for this reason, the dependence of $a_{\mathbf{m n}}$ on $x_{\mathbf{m}}$ is shown explicitly in (4.3). Hence $A$ actually defines a function from $R^{d \times N^{d}}$ to the space of $N^{d} \times N^{d}$ matrices. To emphasize this, we sometimes write $A(\mathbf{x})$ for $A$, where $\mathbf{x}=\left(x_{\mathbf{0}}, \ldots, x_{\mathrm{N}-1}\right)$. Note that $\mathbf{0}, \ldots, \mathbf{N}-\mathbf{1} \in \mathbf{L}$. Define a norm on $\mathcal{T}$ by

$$
|A-B|_{\mathcal{T}}=\sum_{\mathbf{m}, \mathbf{n}}\left|a_{\mathbf{m n}}-b_{\mathbf{m n}}\right| .
$$

Since all the second-order partials of $\Theta_{\sigma}$ are bounded (by numbers depending on $\sigma$ ), and there are only finitely many sigmas $\sigma_{\mathrm{m}}$, we may assume that the first-order partials of the $N^{d}$ functions $D_{\mathbf{m}} \Theta_{\sigma_{\mathbf{m}}}$ are all bounded by a constant. With this, we get, for some constant $K_{1}>0,\left|a_{\mathbf{m n}}\left(x_{\mathbf{m}}\right)-a_{\mathbf{m n}}\left(x_{\mathbf{m}}^{\prime}\right)\right| \leq K_{1}\left|x_{\mathbf{m}}-x_{\mathbf{m}}^{\prime}\right|$, which implies the continuity of $a_{\mathbf{m n}}$ with respect to $x_{\mathbf{m}}$. It follows from (4.4) that

$$
|A(\mathbf{x})-A(\mathbf{y})|_{\mathcal{T}} \leq K_{2} \max _{\mathbf{m}}\left\{\left|x_{\mathbf{m}}-y_{\mathbf{m}}\right|\right\},
$$

where $K_{2}=K_{1} \cdot N^{2 d}$ and $\mathbf{x}, \mathbf{y} \in R^{d \times N^{d}}$. Hence $A(\mathbf{x})$ is continuous in $\mathbf{x}$. We state a standard result regarding the continuity of the inverse of an invertible matrix $A$. Here the continuity is with respect to the elements of $A$.

Proposition 4.1. Let $L\left(R^{n}\right)$ be the set of all $n \times n$ matrices, $\mathcal{I}\left(R^{n}\right)$ the set of $n \times n$ invertible matrices, and $|A|$ the sum of the absolute values of the elements of A. Then,

(1) if $A \in \mathcal{I}\left(R^{n}\right), B \in L\left(R^{n}\right)$, and $|B-A| \cdot\left|A^{-1}\right|<1$, then $B \in \mathcal{I}\left(R^{n}\right)$; and

(2) $\mathcal{I}\left(R^{n}\right)$ is an open subset of $L\left(R^{n}\right)$ and the mapping $A \rightarrow A^{-1}$ is continuous. In fact, if $\left|A^{-1}\right|=\frac{1}{K_{A}}$ and $|B-A|=\beta$, where $\beta<K_{A}$, then $\left|B^{-1}-A^{-1}\right| \leq$ $\frac{\beta}{K_{A}\left(K_{A}-\beta\right)}$.

\footnotetext{
${ }^{1}$ For the sake of simplicity of notation, we use the multi-indexes $\mathbf{m}, \mathbf{n}$, etc., appearing in bold face, as subscripts to denote the elements of a matrix; if we insist on using integer subscripts, we may define a one-to-one $\operatorname{map} k: \mathbf{L} \rightarrow\left\{1, \ldots, N^{d}\right\}$ and write $a_{m n}$ (for the $m n$th element of the matrix $A$ ), where $m=k(\mathbf{m})$ and $n=k(\mathbf{n})$. But we persist with the earlier notation, i.e., bold face subscripting, in order to keep in mind the definition of the elements of the matrices under consideration, as given in (4.3). We follow the same convention for column vectors as well.
} 
Proof. See [14].

Now let for some $\mathbf{x} \in R^{d \times N^{d}}, A=A(\mathbf{x}) \in \mathcal{T}$ be invertible. Then by part 2 of the above proposition, for all invertible matrices $B \in \mathcal{T}$ with $\beta=|B-A|_{\mathcal{T}}<\delta$, where $\delta=\frac{K_{A}}{2}$ ( $K_{A}$ is as in part 2 of the previous proposition), we have

$$
\begin{gathered}
\left|B^{-1}-A^{-1}\right|_{\mathcal{T}} \leq \frac{\beta}{K_{A}\left(K_{A}-\beta\right)} \leq \frac{\beta}{K_{A}\left(K_{A}-\delta\right)}=\frac{2 \beta}{K_{A}^{2}} \\
=\frac{2|B-A|_{\mathcal{T}}}{K_{A}^{2}} .
\end{gathered}
$$

Also, from (4.4), we have $|A(\mathbf{x})-A(\mathbf{y})|_{\mathcal{T}}<\delta$, whenever $\max _{\mathbf{m}}\left\{\left|x_{\mathbf{m}}-y_{\mathbf{m}}\right|\right\}<\delta_{0}=$ $\frac{\delta}{K_{2}}$. Hence, $A(\mathbf{y})$ is invertible. We also have

$$
\left|A^{-1}(\mathbf{y})-A^{-1}(\mathbf{x})\right|_{\mathcal{T}} \leq \frac{2 K_{2}}{K_{A}^{2}} \cdot \max _{\mathbf{m}}\left\{\left|x_{\mathbf{m}}-y_{\mathbf{m}}\right|\right\}
$$

whenever $\left|x_{\mathbf{m}}-y_{\mathbf{m}}\right|<\delta_{0} \forall \mathbf{m}$ for some $\delta_{0}>0$. We summarize this formally in the following theorem.

THEOREM 4.2. Let $A(\mathbf{x}) \in \mathcal{I}_{\mathcal{T}}$, where $\mathcal{I}_{\mathcal{T}}$ is the set of invertible matrices contained in $\mathcal{T}$ and $\mathbf{x} \in R^{d \times N^{d}}$. There is a $\delta_{0}>0$ (depending on $A(\mathbf{x})$ and hence also on $\mathbf{x})$, such that if $\mathbf{y} \in R^{d \times N^{d}}$, and $\max _{\mathbf{m}}\left|x_{\mathbf{m}}-y_{\mathbf{m}}\right|<\delta_{0}$, then $A(\mathbf{y}) \in \mathcal{I}_{\mathcal{T}}$. Moreover,

$$
\left|A^{-1}(\mathbf{y})-A^{-1}(\mathbf{x})\right|_{\mathcal{T}} \leq K \cdot \max _{\mathbf{m}}\left\{\left|x_{\mathbf{m}}-y_{\mathbf{m}}\right|\right\},
$$

where $K>0$ depends on $\delta_{0}$ and $A(\mathbf{x})$.

Finally, we need to know the action of $A^{-1}(\mathbf{y})$ on column vectors as compared to that of $A^{-1}(\mathbf{x})$. For this, we derive an elementary bound on the norms of matrices involving multiplication. Suppose $C_{1}$ and $C_{2}$ are $1 \times n$ column vectors and $A$ and $B$ are $n \times n$ matrices. Then

$$
\begin{gathered}
\left|B C_{2}-A C_{1}\right|=\left|B C_{2}-A C_{2}+A C_{2}-A C_{1}\right| \leq\left|B C_{2}-A C_{2}\right|+\left|A C_{2}-A C_{1}\right| \\
(4.7) \quad \leq|B-A| \cdot\left|C_{2}\right|+|A| \cdot\left|C_{2}-C_{1}\right|,
\end{gathered}
$$

where we choose $|\cdot|$ to be the $\max$-norm in the case of $C_{1}$ and $C_{2}$ and the sum of the absolute values of all the entries in the case of $A$ and $B$.

Let us now combine all the previous results to show the stability of the representation scheme $\mathcal{M}$. Suppose $M, M^{\prime} \in \mathcal{M}$, with $\rho_{\mathcal{M}}\left(M, M^{\prime}\right)=\delta<\delta_{0}$, where $\delta_{0}>0$ will be specified later. Construct the matrix $A_{M}$ as in (4.3), reproduced below for convenience. We have, $A_{M}=\left[a_{\mathbf{m n}}\left(x_{\mathbf{m}}\right)\right], a_{\mathbf{m n}}\left(x_{\mathbf{m}}\right)=D_{\mathbf{m}} \Theta_{\tau_{\mathbf{m}}}\left(x_{\mathbf{m}}-\alpha_{\mathbf{n}}\right)$, where $D_{\mathbf{m}}=\partial_{\mathbf{u}_{m}}$ for some $m$ and $\tau_{\mathbf{m}}=\sigma_{n}$ for some $n$. What we want to show first is that when $\delta_{0}$ is suitably chosen, there is a matrix $A_{M^{\prime}}=A\left(\mathbf{x}^{\prime}\right)$ satisfying the inequality (4.6) with $\mathbf{x}^{\prime}$ in place of $\mathbf{y}$. Next we use the inequality (4.7) to conclude that the representation scheme is stable.

Now since $\rho_{\mathcal{M}}\left(M, M^{\prime}\right)=\delta$, we have $\rho_{\mathcal{H}}\left(M_{j i}, M_{j i}^{\prime}\right) \leq \delta$ for each $i$ and $j$. Thus, in particular, $\rho_{\mathcal{H}}\left(M_{n m}, M_{n m}^{\prime}\right) \leq \delta$. Now by the definition of $\rho_{\mathcal{H}}$, this implies $D\left(M_{n m}, M_{n m}^{\prime}\right) \leq \delta$, where $D$ is as in (4.2). From this, we get $\forall_{\xi \in M_{n m}} \exists_{\zeta \in M_{n m}^{\prime}}$ such that $|\xi-\zeta| \leq \delta$. Note that $\xi, \zeta \in R^{d+1}$. Since the variable $x_{\mathbf{m}}$ appears in $A_{M}$, we must have the relation, $\xi_{\mathbf{m}}=\left(x_{\mathbf{m}}, c_{\mathbf{m}}\right) \in M_{n m}$, where $c_{\mathbf{m}}$ is the $\mathbf{m}$ th entry in the column $C_{M}$ associated with $M$ in our construction. For this $\xi_{\mathbf{m}}$, there is a 
$\zeta_{\mathbf{m}}=\left(x_{\mathbf{m}}^{\prime}, c_{\mathbf{m}}^{\prime}\right) \in M_{n m}^{\prime}$, where $x_{\mathbf{m}}^{\prime} \in R^{d}$ and $c_{\mathbf{m}}^{\prime} \in R$, such that $\left|\xi_{\mathbf{m}}-\zeta_{\mathbf{m}}\right| \leq \delta$; therefore, $\left|x_{\mathbf{m}}-x_{\mathbf{m}}^{\prime}\right| \leq \delta$ and $\left|c_{\mathbf{m}}-c_{\mathbf{m}}^{\prime}\right| \leq \delta$; and this holds for every $\mathbf{m}$. Thus, $\max _{\mathbf{m}}\left\{\left|x_{\mathbf{m}}-x_{\mathbf{m}}^{\prime}\right|\right\} \leq \delta<\delta_{0}$. Now, for $A_{M^{\prime}}$, we choose the matrix $A\left(\mathbf{x}^{\prime}\right)$; and correspondingly, for $C_{M^{\prime}}$, we choose the column vector composed of $c_{\mathbf{m}}^{\prime}$ that appears along with $x_{\mathbf{m}}^{\prime}$ in the previous discussion. It follows that $\left|C_{M^{\prime}}-C_{M}\right| \leq \delta$. All that we need to do is to specify $\delta_{0}$ so that $A\left(\mathbf{x}^{\prime}\right)$ is invertible and satisfies the inequality (4.6). Referring to the discussion prior to Theorem 4.2, we set $\delta_{0}=\frac{1}{2\left|A^{-1}(\mathbf{x})\right| \tau}$. It follows that

$$
\left|A^{-1}\left(\mathbf{x}^{\prime}\right)-A^{-1}(\mathbf{x})\right|_{\mathcal{T}} \leq K \cdot \max _{\mathbf{m}}\left\{\left|x_{\mathbf{m}}^{\prime}-x_{\mathbf{m}}\right|\right\} \leq K \cdot \delta
$$

where $K$ is as in the theorem. Now, referring to the inequality (4.7), with $C_{1}=C_{M}$, $C_{2}=C_{M^{\prime}}, A=A(\mathbf{x})$, and $B=A\left(\mathbf{x}^{\prime}\right)$, we have

$$
\begin{gathered}
\left|A^{-1}\left(\mathbf{x}^{\prime}\right) C_{M^{\prime}}-A^{-1}(\mathbf{x}) C_{M}\right| \leq\left|A^{-1}\left(\mathbf{x}^{\prime}\right)-A^{-1}(\mathbf{x})\right| \mathcal{T}_{\mathcal{T}} \cdot\left|C_{M^{\prime}}\right|+\left|A^{-1}(\mathbf{x})\right| \mathcal{T}^{\cdot}\left|C_{M^{\prime}}-C_{M}\right| \\
\leq K \cdot \delta \cdot\left|C_{M^{\prime}}\right|+\delta \cdot\left|A^{-1}(\mathbf{x})\right|_{\mathcal{T}}=K \cdot \delta \cdot\left(\delta+\left|C_{M}\right|\right)+\frac{\delta}{2 \delta_{0}}=\left(K \cdot\left(\delta+\left|C_{M}\right|\right)+\frac{1}{2 \delta_{0}}\right) \cdot \delta \\
\leq\left(K \cdot\left(\delta_{0}+\left|C_{M}\right|\right)+\frac{1}{2 \delta_{0}}\right) \cdot \delta=\left(K \cdot K_{M}+\frac{1}{2 \delta_{0}}\right) \cdot \rho_{\mathcal{M}}\left(M, M^{\prime}\right),
\end{gathered}
$$

where $K_{M}$ is chosen to be the maximum of all the numbers $\delta_{0}+|c|$ such that for some $x \in R^{d},(x, c) \in M_{j i}$ for some $i$ and $j$. If we put $K_{0}=\left(K \cdot K_{M}+\frac{1}{2 \delta_{0}}\right)$, then the right-hand side of (4.8) becomes $K_{0} \cdot \rho_{\mathcal{M}}\left(M, M^{\prime}\right)$. Now if $M=M(f)$ and $M^{\prime}=M(g)$, where

$$
f=\sum_{\mathbf{m} \in \mathbf{L}} \gamma_{\mathbf{m}} \chi_{I_{\mathbf{m}}} \text { and } g=\sum_{\mathbf{m} \in \mathbf{L}} \lambda_{\mathbf{m}} \chi_{I_{\mathbf{m}}}
$$

then it easy to see that the $\mathbf{m}$ th entry of the column vector $A^{-1}(\mathbf{x}) C_{M}$ is $\gamma_{\mathbf{m}}$ and that of $A^{-1}\left(\mathbf{x}^{\prime}\right) C_{M^{\prime}}$ is $\lambda_{\mathbf{m}}$. Hence $\rho_{\mathcal{S}}(f, g)=\max _{\mathbf{m}}\left|\gamma_{\mathbf{m}}-\lambda_{\mathbf{m}}\right| \leq K_{0} \cdot \rho_{\mathcal{M}}(M(f), M(g))$ for some positive constant $K_{0}$, depending on $M(f)$. All this holds provided, for some $\delta_{0}>0$ (depending on $\left.M(f)\right), \quad \rho_{\mathcal{M}}(M(f), M(g))<\delta_{0}$. We record this result in the following theorem.

THEOREM 4.3 (stability). Let $\mathcal{M}$ be a complete representation, and let $T: \dot{\mathcal{S}} \rightarrow$ $\mathcal{M}$ be the map sending $f$ to $M(f)$. Then for each $M=M(f) \in \mathcal{M}, f \in \dot{\mathcal{S}}$, there are $\delta_{0}, K_{0}>0$ (depending on $M$ ) such that, for every $M^{\prime} \in \mathcal{M}$ with $\rho_{\mathcal{M}}\left(M, M^{\prime}\right)<\delta_{0}$, we have

$$
\rho_{\mathcal{S}}\left(T^{-1}(M), T^{-1}\left(M^{\prime}\right)\right) \leq K_{0} \cdot \rho_{\mathcal{M}}\left(M, M^{\prime}\right)
$$

5. On the continuity of the edge points. In this section, we consider the questions raised at the end of section 2.2. Specifically, let $f_{n} \in \dot{\mathcal{S}}, n=1,2, \ldots$, and $f_{n} \longrightarrow f$, where $f \in \dot{\mathcal{S}}$ as $n \longrightarrow \infty$. Then, the questions, reworded suitably, are as follows:

(1) For each $\xi \in E_{\theta}^{(\sigma)}(f ; \mathbf{u})$, is there a sequence $\left\{\xi_{n}\right\}$ such that $\xi_{n} \in E_{\theta}^{(\sigma)}\left(f_{n} ; \mathbf{u}\right)$ and $\xi_{n} \rightarrow \xi$ as $n \rightarrow \infty$ ?

(2) Do the sets $E_{\theta}^{(\sigma)}\left(f_{n} ; \mathbf{u}\right)$ converge to $E_{\theta}^{(\sigma)}(f ; \mathbf{u})$ as $n \rightarrow \infty$ with respect to any "reasonable" distance measure? 
We answer the first question affirmatively, but the second question is not answered in this work. In what follows, we assume that $D_{i} \Theta_{\sigma}$ and $D_{i j} \Theta_{\sigma}$ are bounded by a real number $K_{\sigma}$, i.e.,

$$
\left|D_{i} \Theta_{\sigma}(x)\right| \leq K_{\sigma} \text { and }\left|D_{i j} \Theta_{\sigma}(x)\right| \leq K_{\sigma}, \quad x \in R^{d}, \quad 1 \leq i, j \leq d
$$

Also, for $f, g \in \mathcal{S}, \xi \in R^{d}$, a standard unit vector $\mathbf{u} \in R^{d}$, all fixed, and $t$ varying over $R$, define

$$
\begin{aligned}
& \phi(t)=\left|\partial_{\mathbf{u}} \tilde{f}_{\sigma}(\xi+t \mathbf{u})\right|^{2}, \\
& \psi(t)=\left|\partial_{\mathbf{u}} \tilde{g}_{\sigma}(\xi+t \mathbf{u})\right|^{2} .
\end{aligned}
$$

Note that since $\tilde{f}_{\sigma}$ is analytic, so also $\phi$; and the same applies to $\psi$. With this, let us start with the following proposition.

Proposition 5.1. Let $f, g \in \mathcal{S}^{1}$, and let $\phi$ and $\psi$ be as given in (5.2) and (5.3), respectively. Then, $\forall t \in R$, the following bound holds:

$$
\left|\phi^{\prime}(t)-\psi^{\prime}(t)\right| \leq 4 K_{\sigma}^{2} N^{2 d} d^{\frac{3}{2}} \rho_{\mathcal{S}}(f, g) .
$$

Proof. By explicitly computing $\phi^{\prime}$, it can be shown (see [3] for details) that

$$
\begin{aligned}
\phi^{\prime}(t) & =\frac{d}{d t}\left(\partial_{\mathbf{u}} \tilde{f}_{\sigma}(\xi+t \mathbf{u})\right)^{2} \\
& =2\left(\sum_{k=1}^{k=d} u_{k} D_{k} \tilde{f}_{\sigma}(\xi+t \mathbf{u})\right) \times\left(\sum_{i=1}^{i=d} \sum_{j=1}^{j=d} u_{i} u_{j} D_{i j} \tilde{f}_{\sigma}(\xi+t \mathbf{u})\right),
\end{aligned}
$$

where $u_{i}$ stands for the $i$ th component of $\mathbf{u}$. Now let

$$
\tilde{f}_{\sigma}(x)=\sum_{\mathbf{m} \in \mathbf{L}} \gamma_{\mathbf{m}} \Theta_{\sigma}\left(x-\alpha_{\mathbf{m}}\right)
$$

Substituting for $\tilde{f}_{\sigma}$ from (5.5) in (5.4), we get

$$
\begin{aligned}
\phi^{\prime}(t)=2( & \left.\sum_{k=1}^{k=d} u_{k} D_{k} \sum_{\mathbf{m} \in \mathbf{L}} \gamma_{\mathbf{m}} \Theta_{\sigma}\left(\xi+t \mathbf{u}-\alpha_{\mathbf{m}}\right)\right) \\
& \times\left(\sum_{i=1}^{i=d} \sum_{j=1}^{j=d} u_{i} u_{j} D_{i j} \sum_{\mathbf{n} \in \mathbf{L}} \gamma_{\mathbf{n}} \Theta_{\sigma}\left(\xi+t \mathbf{u}-\alpha_{\mathbf{n}}\right)\right),
\end{aligned}
$$

which, after rearranging the terms, can be written as

$$
\phi^{\prime}(t)=2 \sum_{i, j, k=1}^{i, j, k=d} \sum_{\mathbf{m}, \mathbf{n} \in \mathbf{L}} u_{k} u_{i} u_{j} \gamma_{\mathbf{m}} \gamma_{\mathbf{n}} D_{k} \Phi_{\mathbf{m}}(t) D_{i j} \Phi_{\mathbf{n}}(t),
$$

where $\Phi_{\mathbf{k}}(t)=\Theta_{\sigma}\left(\xi+t \mathbf{u}-\alpha_{\mathbf{k}}\right), \mathbf{k}=\mathbf{m}, \mathbf{n}$. Throughout this proposition, we follow this notation (i.e., write $\Phi_{\mathbf{k}}(t)$ for $\Theta_{\sigma}\left(\xi+t \mathbf{u}-\alpha_{\mathbf{k}}\right)$ ) in order to make the equations concise. Note that both $D_{i} \Phi_{\mathbf{k}}$ and $D_{i j} \Phi_{\mathbf{k}}$ are bounded by $K_{\sigma}$. Similarly, if $\tilde{g}_{\sigma}(x)=$ $\sum_{\mathbf{m} \in \mathbf{L}} \lambda_{\mathbf{m}} \Theta_{\sigma}\left(x-\alpha_{\mathbf{m}}\right)$, then we have

$$
\psi^{\prime}(t)=2 \sum_{i, j, k=1}^{i, j, k=d} \sum_{\mathbf{m}, \mathbf{n} \in \mathbf{L}} u_{k} u_{i} u_{j} \lambda_{\mathbf{m}} \lambda_{\mathbf{n}} D_{k} \Phi_{\mathbf{m}}(t) D_{i j} \Phi_{\mathbf{n}}(t) .
$$


From (5.6) and (5.7), we get, after considerable simplification (see [3]),

$$
\begin{aligned}
\left|\phi^{\prime}(t)-\psi^{\prime}(t)\right| & =2\left|\sum_{i, j, k=1}^{i, j, k=d} \sum_{\mathbf{m}, \mathbf{n} \in \mathbf{L}} u_{k} u_{i} u_{j}\left(\gamma_{\mathbf{m}} \gamma_{\mathbf{n}}-\lambda_{\mathbf{m}} \lambda_{\mathbf{n}}\right) D_{k} \Phi_{\mathbf{m}}(t) D_{i j} \Phi_{\mathbf{n}}(t)\right| \\
& \leq 2 K_{\sigma}^{2} N^{d} d^{\frac{3}{2}} \times\left(\sum_{\mathbf{m}, \mathbf{n} \in \mathbf{L}}\left|\gamma_{\mathbf{m}} \gamma_{\mathbf{n}}-\lambda_{\mathbf{m}} \lambda_{\mathbf{n}}\right|^{2}\right)^{\frac{1}{2}},
\end{aligned}
$$

where we have used (i) the bounds on $D_{i} \Phi_{\mathbf{k}}$ and $D_{i j} \Phi_{\mathbf{k}}$, given in (5.1); (ii) the Cauchy-Schwartz inequality; and (iii) the fact that $\mathbf{u}$ is a unit vector.

Now,

$$
\begin{aligned}
\left|\gamma_{\mathrm{m}} \gamma_{\mathrm{n}}-\lambda_{\mathrm{m}} \lambda_{\mathrm{n}}\right| & =\left|\gamma_{\mathrm{m}} \gamma_{\mathrm{n}}-\gamma_{\mathrm{m}} \lambda_{\mathrm{n}}+\gamma_{\mathrm{m}} \lambda_{\mathrm{n}}-\lambda_{\mathrm{m}} \lambda_{\mathrm{n}}\right| \\
& \leq\left|\gamma_{\mathrm{m}}\right|\left|\gamma_{\mathrm{n}}-\lambda_{\mathrm{n}}\right|+\left|\lambda_{\mathrm{n}}\right|\left|\gamma_{\mathrm{m}}-\lambda_{\mathrm{m}}\right|
\end{aligned}
$$

Also, since $f, g \in \mathcal{S}^{1}$, we have $\left|\gamma_{\mathbf{k}}\right| \leq 1$ and $\left|\lambda_{\mathbf{k}}\right| \leq 1$, for every $\mathbf{k} \in \mathbf{L}$. Hence the above inequality becomes

$$
\begin{gathered}
\left|\gamma_{\mathrm{m}} \gamma_{\mathbf{n}}-\lambda_{\mathrm{m}} \lambda_{\mathbf{n}}\right| \leq\left|\gamma_{\mathbf{n}}-\lambda_{\mathbf{n}}\right|+\left|\gamma_{\mathrm{m}}-\lambda_{\mathrm{m}}\right| \leq \rho_{\mathcal{S}}(f, g)+\rho_{\mathcal{S}}(f, g) \\
=2 \rho_{\mathcal{S}}(f, g) .
\end{gathered}
$$

Combining (5.9) and (5.8), we get

$$
\begin{array}{r}
\left|\phi^{\prime}(t)-\psi^{\prime}(t)\right| \leq 2 K_{\sigma}^{2} N^{d} d^{\frac{3}{2}} \times\left(\sum_{\mathbf{m}, \mathbf{n} \in \mathbf{L}} 2^{2} \rho_{\mathcal{S}}^{2}(f, g)\right)^{\frac{1}{2}} \\
=2 K_{\sigma}^{2} N^{d} d^{\frac{3}{2}} \times\left(2^{2} \rho_{\mathcal{S}}^{2}(f, g) \times N^{d} \times N^{d}\right)^{\frac{1}{2}} \\
=2 K_{\sigma}^{2} N^{d} d^{\frac{3}{2}} \times\left(2 \rho_{\mathcal{S}}(f, g) N^{d}\right)=4 K_{\sigma}^{2} N^{2 d} d^{\frac{3}{2}} \rho_{\mathcal{S}}(f, g) .
\end{array}
$$

TheOREM 5.2. Let $f, g \in \mathcal{S}^{1}$, and $\xi \in E_{\theta}^{(\sigma)}(f ; \mathbf{u})$. Then for each $\epsilon>0$, there is an $\eta>0$ (depending on $\xi, f$, and $\epsilon$ ) such that

$$
\rho_{\mathcal{S}}(f, g)<\eta \Longrightarrow \exists \zeta \in E_{\theta}^{(\sigma)}(g ; \mathbf{u}) \text { with }|\xi-\zeta|<\epsilon .
$$

Proof. Since $\xi \in E_{\theta}^{(\sigma)}(f ; \mathbf{u})$, there is a $\delta>0$ such that

$$
\left|\partial_{\mathbf{u}} \tilde{f}_{\sigma}(\xi+t \mathbf{u})\right|^{2}<\left|\partial_{\mathbf{u}} \tilde{f}_{\sigma}(\xi)\right|^{2} \text { whenever }|t| \in(0, \delta),
$$

which implies, by (5.2),

$$
\phi(0)>\phi(t) \quad \forall t \text { with } 0<|t|<\delta .
$$

Let $\epsilon^{\prime}=\min \left\{\epsilon, \frac{\delta}{2}\right\}$. So $\epsilon^{\prime}>0$. Now by Rolle's theorem, (5.10) implies that there are $a \in\left(-\epsilon^{\prime}, 0\right)$ and $b \in\left(0, \epsilon^{\prime}\right)$ such that $\phi^{\prime}(a)>0$ and $\phi^{\prime}(b)<0$. Now choose $\eta=\frac{k}{4 K_{\sigma}^{2} N^{2 d} d^{\frac{3}{2}}}$, where

$$
k=\min \left\{\frac{|\phi(a)|}{2}, \frac{|\phi(b)|}{2}\right\}
$$


Let $g \in \mathcal{S}^{1}$ be such that $\rho_{\mathcal{S}}(f, g)<\eta$; and, as before, let $\psi$ be as given in (5.3). By the proposition we have just proved, we have, for each $t \in R$,

$$
\begin{aligned}
\left|\phi^{\prime}(t)-\psi^{\prime}(t)\right| & \leq 4 K_{\sigma}^{2} d^{\frac{3}{2}} N^{2 d} \rho_{\mathcal{S}}(f, g)<4 K_{\sigma}^{2} d^{\frac{3}{2}} N^{2 d} \eta \\
& =4 K_{\sigma}^{2} d^{\frac{3}{2}} N^{2 d} \times \frac{k}{4 K_{\sigma}^{2} N^{2 d}}=k,
\end{aligned}
$$

where $k$ is given by (5.11). So the above inequality reduces to

$$
\left|\phi^{\prime}(t)-\psi^{\prime}(t)\right|<\min \left\{\frac{|\phi(a)|}{2}, \frac{|\phi(b)|}{2}\right\} .
$$

We shall now show that $\psi$ has an edge point $\zeta$ in the interval $(a, b)$. Note that $\psi^{\prime}(a)$ and $\psi^{\prime}(b)$ have the same signs as $\phi^{\prime}(a)$ and $\phi^{\prime}(b)$, respectively. For if $\psi^{\prime}(a)$ and $\phi^{\prime}(a)$ have opposite signs, or if $\psi^{\prime}(a)=0$, then

$$
\left|\phi^{\prime}(a)-\psi^{\prime}(a)\right|=\left|\phi^{\prime}(a)\right|+\left|\psi^{\prime}(a)\right| \geq\left|\phi^{\prime}(a)\right|>\frac{\left|\phi^{\prime}(a)\right|}{2},
$$

contrary to (5.12). Similarly, it can be shown that $\psi^{\prime}(b)$ and $\phi^{\prime}(b)$ have the same sign. So we have $\psi^{\prime}(a)>0$ and $\psi^{\prime}(b)<0$.

Also note that $a<b$, since $a<0$ and $b>0$. Now, by the intermediate value property of $\psi^{\prime}$, there is a point in $(a, b)$ at which $\psi^{\prime}$ vanishes. Also since $\psi^{\prime}$ is nonconstant and analytic on $R$, there are only finitely many zeros in the compact set $[a, b]$. Let these be $t_{1}, \ldots, t_{n}$. It follows that at one of these points, say $t_{j}, \psi$ changes its sign, i.e., there is an $r>0$ such that $\forall t \in\left[t_{j}-r, t_{j}\right), \psi^{\prime}(t)>0$, and $\forall t \in\left(t_{j}, t_{j}+r\right], \psi^{\prime}(t)<0$, implying that $t_{j}$ is a point of local maximum for $\psi$. If we take $\zeta=\xi+t_{j} \mathbf{u}$, then $\zeta \in E_{\theta}^{(\sigma)}(g ; \mathbf{u})$. Also, $|\zeta-\xi|=\left|t_{j}\right|<\max \{|a|,|b|\}<$ $\epsilon^{\prime} \leq \epsilon$.

In the theorem just proved, the requirement that $f, g \in \mathcal{S}^{1}$ may be relaxed. For if $f \in \dot{\mathcal{S}}$ and $c>0$, then $E_{\theta}^{(\sigma)}(f ; \mathbf{u}),=E_{\theta}^{(\sigma)}(c f ; \mathbf{u})$; and if $f=0$, then $E_{\theta}^{(\sigma)}(f ; \mathbf{u})=\emptyset$. Thus we have the following.

Corollary 5.3. Let $f, g \in \mathcal{S}$, and $\xi \in E_{\theta}^{(\sigma)}(f ; \mathbf{u})$. Then for each $\epsilon>0$, there is an $\eta>0$ such that

$$
\rho_{\mathcal{S}}(f, g)<\eta \Longrightarrow \exists \zeta \in E_{\theta}^{(\sigma)}(g ; \mathbf{u}) \text { with }|\xi-\zeta|<\epsilon .
$$

Proof. Fix a $c>0$ such that $\rho_{\mathcal{S}}(c f, 0)<1$. Since $c f$ is an interior point of $\mathcal{S}^{1}$, there is a $\delta_{1}>0$ such that $\rho_{\mathcal{S}}(c f, g)<\delta_{1} \Longrightarrow g \in \mathcal{S}$. By the previous theorem (applied to $c f$ in place of $f$ ), there is a $\delta_{2}>0$ for which the conclusion holds. Let $\delta=\min \left\{\delta_{1}, \delta_{2}\right\}$. Now choose $\eta=\frac{\delta}{c}$. If $\rho_{\mathcal{S}}(f, g)<\eta$, then $\rho_{\mathcal{S}}(c f, c g)<c \eta=\delta$. Hence $c g \in \mathcal{S}^{1}$ and $\rho_{\mathcal{S}}(c f, c g)<\delta_{2}$ which implies, by our choice of $\delta_{2}$, that there is a $\zeta \in E_{\theta}^{(\sigma)}(c g ; \mathbf{u})$ with $|\zeta-\xi|<\epsilon$. Now this $\zeta$ is also in $E_{\theta}^{(\sigma)}(c g ; \mathbf{u})$.

We are now in a position to answer the first of the two questions raised at the beginning of this section. Let $f_{n} \in \dot{\mathcal{S}}$ and $f_{n} \rightarrow f$, where $f \in \dot{\mathcal{S}}$. Then for each point $\xi \in E_{\theta}^{(\sigma)}(f ; \mathbf{u})$, there is a sequence $\xi_{n} \in E_{\theta}^{(\sigma)}\left(f_{n} ; \mathbf{u}\right)$ such that $\xi_{n} \rightarrow \xi$. However, it is not clear whether there is a sequence $\xi_{n} \in E_{\theta}^{(\sigma)}\left(f_{n} ; \mathbf{u}\right)$ such that $\xi_{n} \rightarrow \xi$, but $\xi \notin E_{\theta}^{(\sigma)}(f ; \mathbf{u})$. It is possible that this is true. Experimental evidence for this is found when we add a small amount of noise to a clear image; in this case, we notice 
that extra boundary points are created. While reconstructing, it is better to guard against this possibility by avoiding - or, at least, by reducing - the creation of extra edge points. Based on this observation, we attempt, in the next section, to get a reconstruction that is close in shape to the desired shape. Finally, whether or not there is a possibility of getting extra edge points, it may be of independent interest to know whether the sets $E_{\theta}^{(\sigma)}\left(f_{n} ; \mathbf{u}\right)$ converge to $E_{\theta}^{(\sigma)}(f ; \mathbf{u})$ with respect to any reasonable distance measure ${ }^{2}$ or not. This question is not answered in the present work.

6. Implementation of the reconstruction method. We present a digital version of the reconstruction problem and its solution illustrated by experimental results. In the previous sections, we formulated the problem of RMM, and discussed the theoretical issues of completeness and stability. In our formulation (section 2.2), we introduced a shaper functional and discussed its importance. In this section, we deal explicitly with this.

Although we gave a constructive proof for the existence of a complete representation scheme in section 3, we do not, in practice, resort to this algorithm, for the following reasons:

(1) In the digital version, we discretize the entire space, $R^{d}$, for computational convenience.

(2) When the number of unknowns is large (as is the case with images), we may have to spend considerable computational time in finding the points $x_{n}$ as discussed in the proof of Theorem 3.4.

(3) Numerical stability is different from theoretical stability, as is digital computer arithmetic different from the usual arithmetic. We need to give considerable weight to a regularizing term in order to avoid spurious oscillations and to realize a smooth solution. Hence, even if the representation is complete, we cannot expect to obtain an exact reconstruction.

(4) Finally, with the help of experiments, we attempt to explore how significant the data in terms of geometry are. (This was not taken into account in our theoretical results on completeness and stability.)

We modify the definitions of maxima and employ a representation scheme whose completeness is not guaranteed.

6.1. Discretization of images and modulus maxima. We discuss the implementation of the reconstruction algorithms for images from modulus maxima of the directional derivatives. We assume the directions are along the $x$ - and $y$-axes.

Suppose $\gamma$ is an $N \times N$ matrix. We denote the convolved version at scale $\sigma$ with $\gamma_{\sigma}$. Therefore, we write $\gamma_{\sigma}(i, j)=\tilde{f}_{\sigma}\left(\frac{i}{N}, \frac{j}{N}\right)$. Note that the convolution is in two variables in this case; hence if we expand the above, we get a messy expression for the relationship between $\gamma$ and $\gamma_{\sigma}$. To simplify matters, we assume that $\theta$ is variables-separable, i.e., $\theta(x, y)=\varphi(x) \varphi(y)$ for some $\varphi$ defined over $R$. It follows that $\Theta_{\sigma}(x, y)=\phi_{\sigma}(x) \phi_{\sigma}(y)$, where

$$
\phi_{\sigma}(x)=\int_{0}^{\frac{1}{N}} \frac{1}{\sigma} \varphi\left(\frac{x-\xi}{\sigma}\right) d \xi .
$$

Hence, we can write in the matrix form the relationship between $\gamma_{\sigma}$ and $\gamma$ as follows: $\gamma_{\sigma}=H_{\sigma} \gamma H_{\sigma}^{T}$, where $H_{\sigma}$ is the $N \times N$ matrix whose $i j$ th component is $\phi_{\sigma}\left(\frac{j-i}{N}\right)$.

\footnotetext{
${ }^{2} \mathrm{~A}$ distance measure that one can try may be the Hausdorff metric restricted to the set of the closures of the sets $E_{\theta}^{(\sigma)}(g ; \mathbf{u}) \bigcap B(0, r)$ for some $r>0$.
} 
Note that in the case of boundary points, we are concerned with the partial derivatives. It is easy to see that the partials take the form

$$
D_{1} \gamma_{\sigma}=G_{\sigma} \gamma H_{\sigma}^{T} \text { and } D_{2} \gamma_{\sigma}=H_{\sigma} \gamma G_{\sigma}^{T},
$$

where $G_{\sigma}$ is the matrix whose $i j$ th entry is $\phi_{\sigma}^{\prime}\left(\frac{j-i}{N}\right)$.

We assume that we are given the following input data: (i) the row modulus maxima points of the matrix $D_{1} \gamma_{\sigma}$, along with entries of the matrix $D_{1} \gamma_{\sigma}$ at these locations, and (ii) the column modulus maxima points of the matrix $D_{2} \gamma_{\sigma}$, along with the entries of the matrix $D_{2} \gamma_{\sigma}$ at these locations. Let the row modulus maxima locations of $D_{1} \gamma_{\sigma}$ be $k_{i j}, i=0, \ldots, N-1, j=1, \ldots, m_{i}$, and the column modulus maxima locations of $D_{2} \gamma_{\sigma}$ be $l_{i j}, j=0, \ldots, N-1, i=1, \ldots, n_{j}$. With this information, we proceed to reconstruct in two stages. In the first stage, we construct approximations $h_{1}$ and $h_{2}$ to $D_{1} \gamma_{\sigma}$ and $D_{2} \gamma_{\sigma}$, respectively; and in the second stage, using these approximations, we construct a regularized solution by minimizing a cost functional of the form

$$
\begin{gathered}
\Omega(\lambda)=\Omega_{1}(\lambda)+\Omega_{2}(\lambda), \\
\Omega_{1}(\lambda)=c_{1}\left\|L_{1} \lambda\right\|^{2}+\left\|D_{1} \lambda_{\sigma}-h_{1}\right\|^{2}, \\
\Omega_{2}(\lambda)=c_{2}\left\|\lambda L_{2}\right\|^{2}+\left\|D_{2} \lambda_{\sigma}-h_{2}\right\|^{2},
\end{gathered}
$$

where $L_{1}$ and $L_{2}$ are the regularization operators. In our experiments, we choose $L_{1}$ to be the forward difference operator on the rows and $L_{2}$ to be the forward difference operator on the columns.

6.2. Heuristics for $D_{1} \gamma_{\sigma}$ and $D_{2} \gamma_{\sigma}$. We present first a heuristic $h_{1}$ for $D_{1} \gamma_{\sigma}$. Later, we construct one for $D_{2} \gamma_{\sigma}$ with minor modifications.

Suppose $k_{i j}, i=0, \ldots, N-1, j=1, \ldots, m_{i}$, are the row modulus maxima points of $D_{1} \gamma_{\sigma}$. We know the values $D_{1} \gamma_{\sigma}\left(i, k_{i j}\right)$; hence, we need to interpolate (or extrapolate) for the values of $D_{1} \gamma_{\sigma}(i, n)$ only for $n \neq k_{i j}$. In the process, we must somehow take into account the fact that $k_{i j}$ are the modulus maxima locations of $D_{1} \gamma_{\sigma}$. We achieve this by choosing a suitable interpolating function so that this may result in creating modulus maxima at these locations only.

Let $\psi: R \rightarrow R$ be continuously differentiable having the following properties:

(1) $\psi(x) \geq 0, \psi(0)=1, \lim _{|x| \rightarrow \infty} \psi(x)=0$, and

(2) $x \psi^{\prime}(x) \leq 0$.

More explicitly, property 2 above implies $\psi$ decreases to 0 as $x \rightarrow \infty$ and as $x \rightarrow-\infty$. We use $\psi$ as our interpolating function and define

$$
h_{1}(i, n)=\sum_{j=1}^{j=m_{i}} D_{1} \gamma_{\sigma}\left(i, k_{i j}\right) \psi\left(\frac{n-k_{i j}}{N}\right) .
$$

Of course, we may not have $h_{1}\left(i, k_{i j}\right)=D_{1} \gamma_{\sigma}\left(i, k_{i j}\right)$; but if we choose $\psi$ with fast decay, then the two quantities are nearly equal (provided the locations $k_{i(j-1)}, k_{i j}$, and $k_{i(j+1)}$ are sufficiently far away from one another). Also, our purpose is only to get a solution similar in shape to $D_{1} \gamma_{\sigma}$ rather than get the exact values at these locations. Otherwise, we may choose

$$
h_{1}(i, n)=\sum_{j=1}^{j=m_{i}} \beta_{j} \psi\left(\frac{n-k_{i j}}{N}\right),
$$


where $\beta_{j}$ are evaluated by requiring $h_{1}\left(i, k_{i j}\right)=D_{1} \gamma_{\sigma}\left(i, k_{i j}\right)$, as in the case of interpolation using radial basis functions [15]. But this will lead to a number of matrix inversions. In contrast, the evaluation in (6.3) is very simple and does not involve any matrix inversions.

Finally, for obtaining the heuristic $h_{2}$ for $D_{2} \gamma_{\sigma}$, we modify the interpolation scheme in (6.3) as follows:

$$
h_{2}(n, j)=\sum_{i=1}^{i=n_{j}} D_{2} \gamma_{\sigma}\left(l_{i j}, j\right) \psi\left(\frac{n-k_{i j}}{N}\right) .
$$

For the purpose of illustration, with the help of experimental results, we choose $\theta(x, y)=\varphi(x) \varphi(y)$, where

$$
\varphi(x)=\frac{e^{-x}}{\left(1+e^{-x}\right)^{2}} .
$$

Note that, except for a factor of $2, \varphi$ is itself a candidate for $\psi$, the interpolating function. However, we choose $\psi$ as

$$
\psi(x)=\frac{2 e^{-x / \tau}}{\left(1+e^{-x / \tau}\right)^{2}},
$$

where $\tau$ can be chosen depending on $\sigma$. With this choice of $\psi$, if we get $h_{1}$ from (6.3) with $\tau=\tau_{1}$, and $h_{2}$ from (6.4) with $\tau=\tau_{1}$, then the parameters implicitly involved in $\Omega$ are $\tau_{1}, \tau_{2}, c_{1}$, and $c_{2}$, so that with $\nu=\left(\tau_{1}, \tau_{2}, c_{1}, c_{2}\right)$, we have $\omega_{\nu}=\Omega$, provided we can demonstrate that $\Omega$ goes to $\mu$, defined in (2.5), as $\tau_{i}, c_{i} \rightarrow 0$. It is easy to see that, by our choice of $\psi$, as $\tau_{i} \rightarrow 0, h_{i}$ degenerate to the matrices $h_{i}^{\prime}$, whose entries are zeros except at the respective maxima locations where the entries are the values of the corresponding derivatives. Finally, we note that, substituting $h_{i}^{\prime}$ in place of $h_{i}$ and letting $c_{i}=0$, the expression for $\Omega$ in (6.2) yields $\mu$.

6.3. Experimental results. The experimental results are shown in Figures 14 , the detailed description of which is given below. We have used the values $\sigma=0.9$ and $\tau=0.85$.

(1) Figure 1 shows the original image.

(2) The two images in Figure 2 correspond to the edge points (at scale 0.9) in the standard $x$ - and $y$-directions corresponding to the original image. Observe that the edge points along a particular direction, $\mathbf{u}$, are aligned in the direction perpendicular to $\mathbf{u}$. The total number of edge points (shown in both the images) is approximately 2000, whereas Parrot itself is a $128 \times 128$ image.

(3) The two images in Figure 3 correspond to the heuristic solutions $h_{1}$ and $h_{2}$. Observe that there are patches along the direction of interpolation, $\mathbf{u}$; this is because of loss of continuity along the direction perpendicular to $\mathbf{u}$. To get around this, we employ regularizing terms leading to cost functionals $\Omega_{i}$ as in (6.2). The images shown are actually obtained by minimizing the corresponding cost functionals with regularizing coefficients $c_{1}=c_{2}=0.6$. Note that a further increase in the regularizing coefficients results in an excessive blurring of the reconstructed images (which, we observe, are already blurred).

(4) The image in Figure 4 is the reconstruction obtained by minimizing $\Omega$ in (6.2). In place of $h_{1}$ and $h_{2}$ in the expression for $\Omega$, we have used the solutions shown in Figure 3, in the corresponding order. We observe that (i) the 


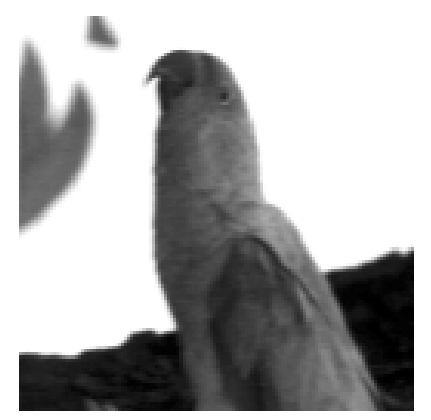

FIG. 1. Parrot. Original image.
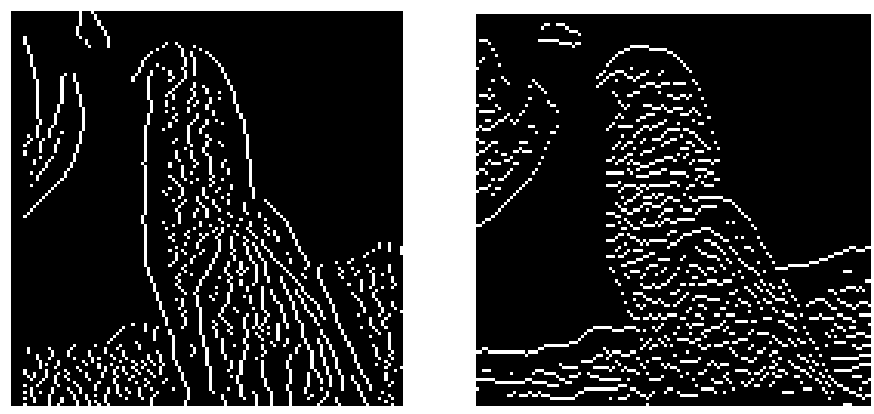

FIG. 2. Edge points. Left, $x$-direction; right, $y$-direction.
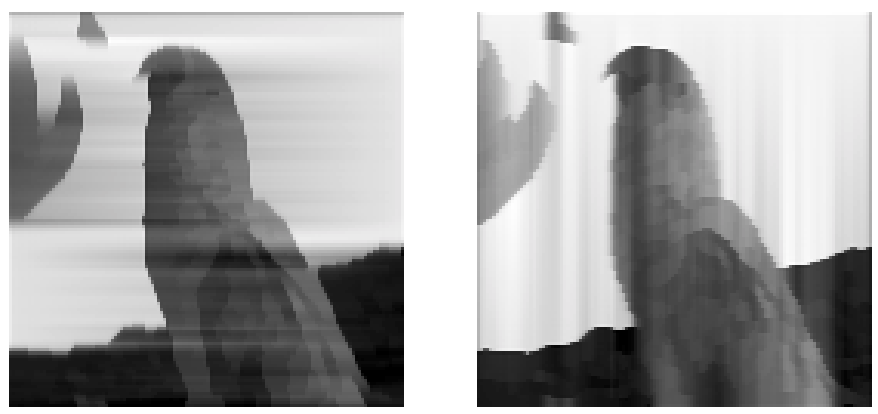

FIG. 3. Heuristic solutions. Left, by minimizing $\Omega_{1}$; right, by minimizing $\Omega_{2}$.

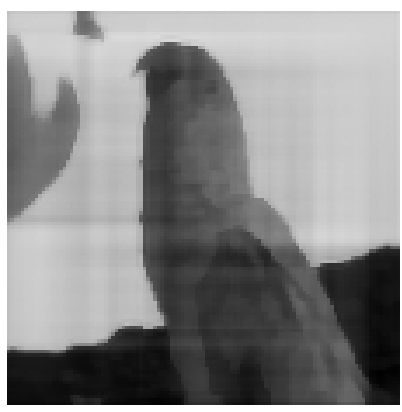

FIG. 4. Reconstructed image. 
reconstructions are slightly blurred and (ii) some of the finer details are lost. It is very difficult to make up for (ii), since the data used for reconstruction do not contain information at this fine level.

7. Conclusions. The present work deals with a certain class of inverse problems, exemplified by reconstruction of signals/images from (i) zero crossings; (ii) Fourier sign information; (iii) extrema; and (iv) modulus maxima.

As applied to image reconstruction, the data used for representation are the locations of the modulus maxima of the directional derivatives, with the corresponding values of the derivatives as auxiliary information.

A large part of the relevant literature that we have surveyed aims at establishing conditions under which a one-dimensional signal or an image is determined uniquely by the information regarding the zero crossings. Observe that the following two problems are different:

(1) Given a class $\mathcal{S}$ of signals, can we define a complete and stable representation $\mathcal{M}$ for the elements of $\mathcal{S}$ ?

(2) Given a representation $\mathcal{M}$ of some class, $\mathcal{S}$, of signals, for what subclass $\mathcal{S}^{\prime} \subset \mathcal{S}$ is the representation complete and stable?

In image analysis, we are at liberty to choose our representation, for the simple reason that we know a priori the original image. If we know only the representation, not the original, then we may need to consider the second question. However, in the present work, our attempt was to answer the first question as applied to image analysis. In the literature, the problem of zero crossings arises in different contexts (see the introductory section of [7]). But it is not clear why the problem of reconstruction from the boundaries is posed as a problem of reconstruction from zero crossings, in spite of the facts that (i) as mentioned earlier (at the beginning of the literature survey), this is probably because of an early work reported in [4] and (ii) that there are explicit attempts to justify this approach (see [2]). The difficulty in working with the zero crossings (or sign data) is, to quote from [7], "...unique reconstruction is not ensured when the sign data are sampled"; and so, stability is a very important issue.

We have formulated the problem of reconstruction from boundaries taking into account the nature of the partial information available. To this end, we assume that in addition to the boundary points, the value of the directional derivative at each of these points is specified. In the literature, it is a common practice to use gradient information at the boundary points for segmentation of images. Therefore, as applied to our representation, there is no overhead if we use the gradient as auxiliary information.

We have presented a new framework for representing multidimensional signals employing certain partial information, namely, (i) points at distance less than $\epsilon$ away from edge points and (ii) the value of the directional derivative at each of the points. This modification was developed from two thoughts: (i) we should allow the data to be sampled and (ii) we should be able to demonstrate completeness of the information if care is taken while sampling the data. We have shown that the partial information becomes complete under certain reasonable conditions. The proof of completeness is constructive (in fact, algorithmic). Throughout the development of the theory, we have taken care to maintain the original space of signals as a complete vector space. This is a very important point, since addition is a fundamental operation. Later, we presented a proof of stability of a complete representation scheme. While developing the theory, however, we have not taken into account the geometrical significance of the information. Finally, we have attempted to show that the edge points of an image 
are perturbed by small distances when the original undergoes a small perturbation.

The important point is that we have kept the formulation of reconstruction problem separate from the theory: whether or not any of the conditions we discussed in the theoretical part hold, we do produce a reconstruction. In fact, we do not even check whether a representation is complete in practice. In our experimental results, we have attempted to take into account the geometrical significance of the data, by introducing a shaper functional. In the process, we have developed algorithms specifically keeping in view the difficulties associated with the arithmetic on a digital computer. Here, we have laid more emphasis on obtaining a solution that is close in shape to the original.

But the basic questions, raised at the end of the introductory section, are not answered in this work. The questions, to repeat, are these:

(1) Do the sets $\left\{E_{\theta}^{\sigma}(f), \sigma>0\right\}$ or $\left\{Z_{\theta}^{\sigma}(f), \sigma>0\right\}$ characterize $f$ completely?

(2) If the answer to the above question is negative, then what conditions are to be imposed on $f$ and/or what additional information regarding $f$ is required so as to elicit an affirmative answer?

(3) If the answer is affirmative (possibly after imposing suitable conditions on $f$ and/or invoking additional information), then is there a stable algorithm to retrieve $f$ ?

For several other interesting questions, see [3]. To summarize, we have, in the present work, introduced some new ideas for dealing with one of the most important problems in computer vision, namely the reconstruction of images from boundaries. These ideas, we hope, will facilitate answers to the above questions.

\section{REFERENCES}

[1] D. Marr, Early processing of visual information, Philos. Trans. Roy. Soc. London Ser. B, 275 (1976), pp. 483-518.

[2] J. J. Clark, Authenticating edges produced by zero-crossing algorithms, IEEE Trans. PAMI, 11 (1989), pp. 43-57.

[3] D. Ravi and Y. V. Venkatesh, A New Framework for Reconstruction of Images from Boundaries, Technical Report 1, Department of Electrical Engineering, Indian Institute of Science, Bangalore, India, 1998.

[4] B. F. LogAN, Information in the zero crossings of bandpass signals, Bell Sys. Tech. J., 56 (1977), pp. 487-510.

[5] D. Rotem AND Y. Y. ZeEvi, Image reconstruction from zero crossings, IEEE Trans. ASSP, 34 (1986), pp. 1269-1277.

[6] S. R. Curtis, A. V. Oppenheim, And J. S. Lim, Signal reconstruction from Fourier transform sign information, IEEE Trans. ASSP, 33 (1985), pp. 643-657.

[7] J. L. SANZ, Multidimensional signal representation by zero crossings: An algebraic study, SIAM J. Appl. Math., 49 (1989), pp. 281-295.

[8] J. L. SANZ AND T. T. HuAng, Image representation by sign information, IEEE Trans. PAMI, 11 (1989), pp. 729-738.

[9] J. L. Sanz AND T. S. Huang, Stability of unique Fourier transform reconstruction, J. Opt. Soc. Amer., 73A (1983), pp. 1442-1445.

[10] R. Hummel and R. Moniot, Reconstructions from zero crossings in scale space, IEEE Trans. ASSP, 37 (1989), pp. 2111-2130.

[11] S. Mallat, Zero-crossings of a wavelet transform, IEEE Trans. Inform. Theory, 37 (1991), pp. 1019-1033.

[12] S. Mallat and S. Zhong Characterization of signals from multiscale edges, IEEE Trans. PAMI, 14 (1992), pp. 710-732.

[13] A. N. Tikhonov and V. Y. Arsenin, Solution of Ill-posed Problems, J. Fritz, trans. ed., V. H. Winston, Washington D.C., 1977.

[14] W. Rudin, Principles of Mathematical Analysis, 3rd ed., McGraw-Hill, New York, 1976, pp. 209.

[15] M. J. D. Powell, The theory of radial basis function approximation in 1990, in Adv. Numer. Anal. 2, Oxford University Press, New York, 1992, pp. 105-210. 